Research Article

\title{
The Preventive Effect of Cardiac Sympathetic Denervation Induced by 6-OHDA on Myocardial Ischemia-Reperfusion Injury: The Changes of lncRNA/circRNAs-miRNA-mRNA Network of the Upper Thoracic Spinal Cord in Rats
}

\author{
Zhixiao Li, ${ }^{1}$ Yujuan Li, ${ }^{1}$ Zhigang He, ${ }^{1}$ Zhen Li, $^{1}$ Weiguo Xu $\mathbb{D}^{2},{ }^{2}$ and HongBing Xiang $\mathbb{D}^{1}$ \\ ${ }^{1}$ Department of Anesthesiology and Pain Medicine, Tongji Hospital of Tongji Medical College, Huazhong University of Science \\ and Technology, Wuhan, 430030 Hubei, China \\ ${ }^{2}$ Department of Orthopedics, Tongji Hospital of Tongji Medical College, Huazhong University of Science and Technology, \\ Wuhan 430030, China
}

Correspondence should be addressed to Weiguo Xu; 354818938@qq.com and HongBing Xiang; xhbtj2004@163.com

Received 17 July 2021; Accepted 13 September 2021; Published 29 November 2021

Academic Editor: Xin Yi

Copyright (c) 2021 Zhixiao Li et al. This is an open access article distributed under the Creative Commons Attribution License, which permits unrestricted use, distribution, and reproduction in any medium, provided the original work is properly cited.

In this study, we investigated whether chemical 6-hydroxydopamine (6-OHDA) stimuli caused cardiac sympathetic denervation (SD), and we analyzed gene expression profiles to determine the changes in the lncRNA/circRNAs-miRNA-mRNA network in the affected spinal cord segments to identify putative target genes and molecular pathways in rats with myocardial ischemiareperfusion injury (MIRI). Our results showed that cardiac sympathetic denervation induced by 6-OHDA alleviated MIRI. Compared with the ischemia reperfusion (IR, MIRI model) group, there were 148 upregulated and 51 downregulated mRNAs, 165 upregulated and 168 downregulated lncRNAs, 70 upregulated and 52 downregulated circRNAs, and 12 upregulated and 11 downregulated miRNAs in the upper thoracic spinal cord of the SD-IR group. Furthermore, we found that the differential genes related to cellular components were mainly enriched in extracellular and cortical cytoskeleton, and molecular functions were mainly enriched in chemokine activity. Pathway analysis showed that the differentially expressed genes were mainly related to the interaction of cytokines and cytokine receptors, sodium ion reabsorption, cysteine and methionine metabolism, mucoglycan biosynthesis, cGMP-PKG signaling pathway, and MAPK signaling pathway. In conclusion, the lncRNA/circRNAsmiRNA-mRNA networks in the upper thoracic spinal cord play an important role in the preventive effect of cardiac sympathetic denervation induced by 6-OHDA on MIRI, which offers new insights into the pathogenesis of MIRI and provides new targets for MIRI.

\section{Introduction}

Myocardial ischemia/reperfusion injury (MIRI) accounts for a large proportion of the total incidence of heart diseases, and it seriously affects human quality of life [1-3]. Previous studies have reported the cellular and molecular mechanisms of neural-cardiac interactions $[4,5]$ during pathological remodeling after MIRI [6]. However, to date, no effective methods have been found to prevent MIRI.

Cardiac nerves, comprising both the sensory nerves and the autonomic nerves, transmit the information from the heart to the spinal cord and brain, which then results in an appropriate sympathetic neural outflow [7]. The role of sympathetic activity in the development of cardiac electrical activity has been well known for decades [8, 9]. Neural regulation is involved in an imbalance between the sympathetic and parasympathetic nervous systems within the ischemic myocardial tissues. Experimental studies have shown that cardiac innervation abnormality is an important cause of the sympathetic nervous system overactivity. Several studies have reported the vital role of the sympathetic nerves in MIRI progression, and sympathetic nerves have been shown 
to infiltrate the myocardial microenvironment thereby accelerating cardiac injury $[10,11]$. In this study, we examined the preventive effect of cardiac sympathetic denervation induced by 6-OHDA, a catecholamine-specific toxin [12], on MIRI rats.

Myocardial ischemia/reperfusion injury is a complex process, and further understanding of the related biological processes and their regulation is necessary [13, 14]. Several lines of evidence have revealed a close correspondence between the spinal cord and cardiovascular system [15-17]; indeed, the spinal cord and cardiovascular system develop in concert and are functionally interconnected in heart disease. It is an accepted fact that noncoding RNAs (ncRNAs) of the spinal cord are vital components of the regulation and cross-talk among MIRI-related signaling pathways [16]. In recent years, a strong consensus has been reached that ncRNAs, including long noncoding RNAs (lncRNAs) and circular RNAs (circRNAs), play an important role in many cellular processes and occurrence of diseases [18-23]. However, the underlying mechanisms based on the function of lncRNAs, circRNAs, and mRNAs in the spinal cord following MIRI remain unclear. Thus, it is necessary to analyze the lncRNAs and circRNAs comprehensively and explore the role of the lncRNA/circRNAs-miRNA-mRNA network in MIRI.

In this study, we first investigated whether cardiac sympathetic denervation induced by 6-OHDA alleviates MIRI. Next, we performed high-throughput sequencing on the spinal cord tissues for the first time to describe and analyze the expression profiles of ncRNAs, including lncRNA and circRNA. Furthermore, we performed Gene Ontology (GO) and Kyoto Encyclopedia of Genes and Genomes (KEGG) analysis of differentially expressed lncRNAs and circRNAs. We also constructed lncRNA/circRNA-miRNA-mRNA networks to further explore their underlying mechanism and possible relationships in the preventive effect of cardiac sympathetic denervation induced by 6-OHDA on MIRI.

\section{Materials and Methods}

2.1. Animals. Adult male Sprague-Dawley rats (weighing 250-300 g) were used, and two animals were placed in each cage. The animals had free access to food and water and were housed in a light- and temperature-controlled room. The experiment started following the approval of the Institutional Animal Care and Use Committee in Tongji Hospital, Huazhong University of Science and Technology (approval No. TJ-A0804).

2.2. Groups and Chemical Sympathetic Denervation. The rats were randomly assigned to the following two groups: MIRI model (IR) group and sympathetic denervation (SD) + IR (SD-IR) group ( $n=9$ for each group). In the SD-IR group, intraperitoneal (i.p.) injections of $50 \mathrm{mg} / \mathrm{kg}$ 6-hydroxydopamine (6-OHDA, Sigma), containing $0.1 \%$ ascorbic acid in the saline solution of 6-OHDA, were administered for 3 consecutive days [1, 24, 25], whereas MIRI rats received i.p. injections of the same volume of saline. One day after the last injection, rats $(n=6$ for each group) were
TABle 1: Primer sequences for reverse transcriptase-quantitative polymerase chain reaction.

\begin{tabular}{lc}
\hline Primer name & Primer sequences $\left(5^{\prime}\right.$ to $3^{\prime}$ ) \\
\hline LncRNA & \\
NONRATT012797.2-F & CTGGGGTGAGAAGGGCTGAC \\
NONRATT012797.2-R & AAGGTGTTTTCCCGGAGGGC \\
NONRATT029190.2-F & ACTGGGGTGGCACTTAGAGG \\
NONRATT029190.2-R & TGTCCACCGTAACATCCCCT \\
NONRATT000247.2-F & CAGGGCCTTGTGCTTGCTA \\
NONRATT000247.2-R & ATGTTTTCCCTCCGCTGCTT \\
NONRATT004098.2-F & ACCTCTTCCCCTCAGCCTACAG \\
NONRATT004098.2-R & TCACTGCCATGAATCACATTCCA \\
NONRATT025664.2-F & ATGCCAACCTTACTATACGTTTCC \\
NONRATT025664.2-R & TGACTCTCCCACCAACTTCAG \\
mRNA & \\
Ubd-F & GGTGAAGCCCAGTGATGAAGAGC \\
Ubd-R & GGGAGGCACAGCAGTCACATTC \\
Ccl12-F & CTGCTCATAGCTGCCGCCATC \\
Ccl12-R & GCCTCCGAATGTGGATCTTCTGC \\
Cxcl10-F & GTTCTCTGCCTCGTGCTGCTG \\
Cxcl10-R & AACATGCGGACAGGATAGACTTGC \\
LOC100912599-F & GCAGTTCAAGCAGCAGCATCAC \\
LOC100912599-R & AACAAGGGACACCATTCACAGAGC \\
Dpep1-F & CATCGCATGTGCCAGCTCTATCC \\
Dpep1-R & GCCACCTTCCACGCCAATCAG \\
GAPDH-F & AGCCCAGGATGCCCTTTAGT \\
GAPDH-R & \\
\hline &
\end{tabular}

deeply anesthetized, and left ventricular tissues were harvested for further laboratory study, whereas other rats received the establishment of MIRI model.

2.3. Establishment of the MIRI Model. The MIRI model was modified from a previous study $[3,15,16,26,27]$. In brief, after routine disinfection, anesthesia, and tracheal intubation, surgical thoracotomy was conducted. In both groups ( $n=9$ for each group), the left anterior descending coronary artery (LAD) was ligated $2 \mathrm{~mm}$ below the left atrial appendage for 30 minutes and then reperfused for 2 hours. The core temperature was maintained throughout the protocol. The rats were monitored to confirm ischemic ST segment elevation during LAD occlusion by an electrocardiogram. Serum troponin cTnl of the two groups was measured 2 hours after reperfusion as an index of myocardial necrosis. Hearts were harvested for hematoxylin and eosin (H\&E) staining and 2,3,5-triphenyl tetrazolium chloride (TTC) staining.

2.4. Determination of Norepinephrine Content. The norepinephrine (NE) content of myocardial tissue from the left ventricle was measured using a high-performance liquid chromatography (HPLC) method [28-31]. In brief, cardiac tissue of the left ventricle was weighed (about $100 \mathrm{mg}$ ) and homogenized in $500 \mu \mathrm{L}$ of precooled methanol/water $(\mathrm{V}: \mathrm{V}=2 / 1)$. The homogenate mixture was sonicated 


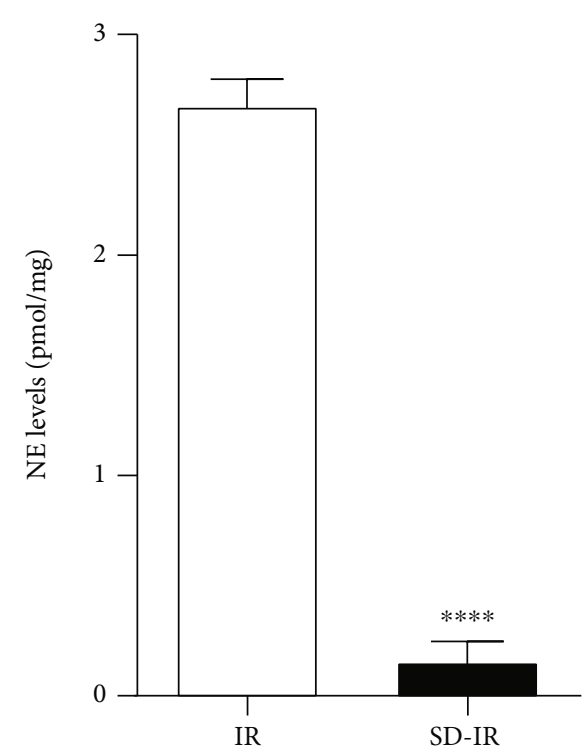

(a)

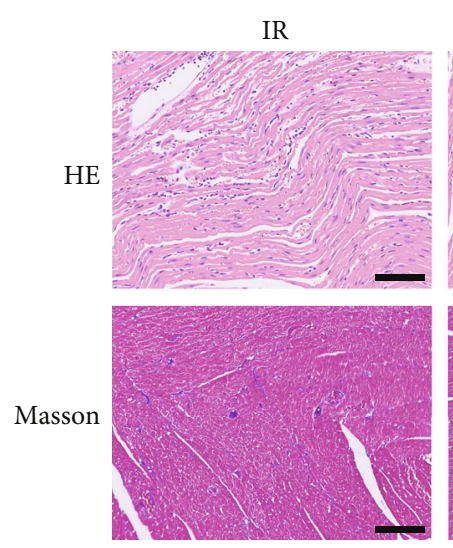

(c)

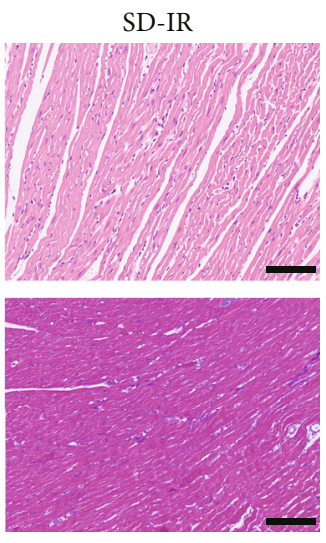

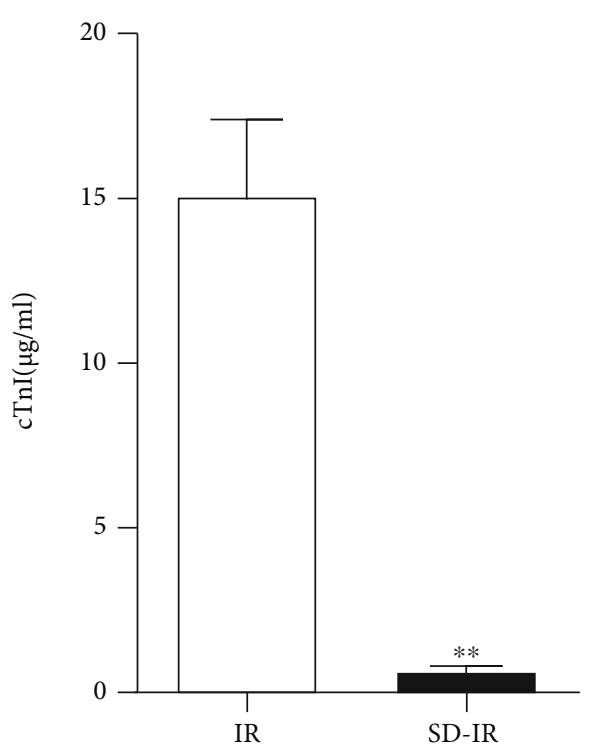

(b)
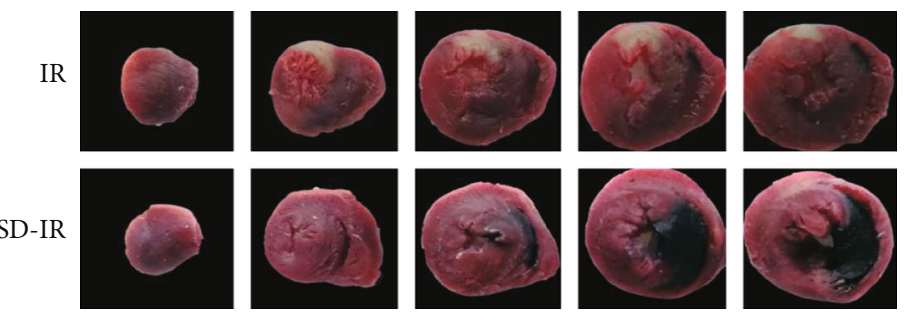

(d)

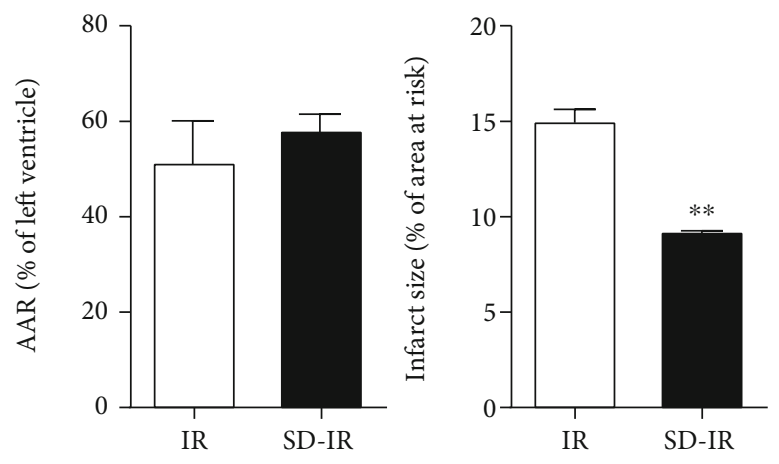

(e)

Figure 1: Chemical sympathetic denervation attenuates myocardial ischemia-reperfusion injury. (a) Serum NE and (b) serum cTnI concentrations in rats with IR or SD-IR. (c) Representative images of hematoxylin and eosin staining and Masson trichrome staining of rat hearts $24 \mathrm{~h}$ after IR injury. Scale bar $=100 \mu \mathrm{m}$. (d) Representative photographs of TTC-Evan blue staining in hearts subjected to IR and SD-IR surgery. (e) Quantification of AAR and infarct area vs. AAR in rat hearts in IR group and SD-IR group. Data are expressed as mean \pm SEM. $* * p<0.01, * * * * p<0.0001$ vs. IR group. NE: norepinephrine; IR: ischemia reperfusion; AAR: area at risk.

(12000 rpm, 10 minutes, $4^{\circ} \mathrm{C}$ ) and the supernatant was collected, while the remaining pellets were repeatedly treated twice. Three supernatants were combined and dried, and then redissolved in $100 \mu \mathrm{L}$ formic acid solution (0.1\%). Next, the following chemicals were added in turn to the supernatant $(10 \mu \mathrm{L})$ : NEM solution $(2.5 \mathrm{mM}, 80 \mu \mathrm{L})$, tBBT solution $(1 \mathrm{M}$ in DMSO, $10 \mu \mathrm{L})$, borate buffer $(0.2 \mathrm{M}, \mathrm{pH} 8.8$, $700 \mu \mathrm{L})$, 5-AIQC solution $(200 \mu \mathrm{L})$, formic acid $(10 \mu \mathrm{L})$. 


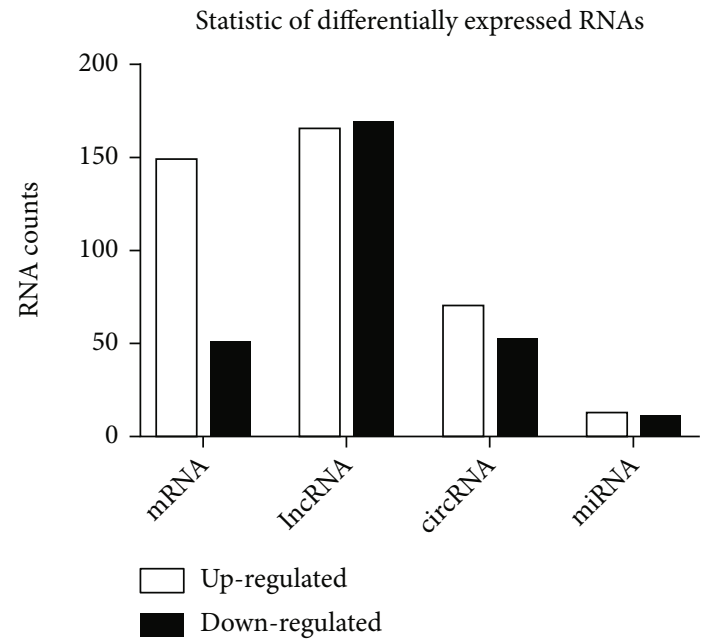

Figure 2: Differentially expressed RNAs. Through highthroughput RNA sequencing, we found that 148 mRNAs were upregulated and $51 \mathrm{mRNAs}$ were downregulated; $165 \operatorname{lncRNAs}$ were upregulated and $168 \operatorname{lncRNAs}$ were downregulated; 70 circRNAs were upregulated and 52 circRNAs downregulated; and 12 miRNAs were upregulated and 11 miRNAs were downregulated.

The solution was sonicated (12000 rpm, 10 minutes, $\left.4^{\circ} \mathrm{C}\right)$ and the supernatant was filtered by a $0.22 \mu \mathrm{m}$ membrane filter before HPLC analysis. HPLC analyses were conducted on the 1290-6470 UPLC-MS/MS system (Agilent, USA). Data preprocessing was performed using Mass Hunter Workstation software (Agilent, Version B.08.00).

2.5. Myocardial Tissue Staining. Myocardial tissue staining was performed as described previously [15]. In brief, each myocardial tissue sample was cut transversely. Hematoxylin and eosin (H\&E) staining was used to observe myocardial pathology. After deparaffinization, $4-\mu \mathrm{m}$-thick sections were immersed in hematoxylin (cat. no. H9627; Sigma-Aldrich; Merck KGaA) for 5-7 min at room temperature, differentiated in $1 \%$ acid alcohol for 2-5 seconds, and stained with $0.5 \%$ eosin (cat. no. 71014544; Sinopharm Chemical Reagent Co., Ltd.) for 2 minutes at room temperature.

After rinsing with distilled water for 30 seconds, the sections were dehydrated with graded alcohols and cleared in xylene. Infarct size was assessed by TTC staining 2 hours after reperfusion. After surgery, the hearts were removed and frozen for 20 minutes at $-20^{\circ} \mathrm{C}$, and then transversally cut into sections with a thickness of $1-2 \mathrm{~mm}$. The tissue sections were incubated for 10 minutes in 2\% TTC in dark conditions at $37^{\circ} \mathrm{C}$ and then fixed overnight in $10 \%$ formaldehyde at $4^{\circ} \mathrm{C}$. The infarct area was white, while the normal tissues were red.

Infarct size and area at risk (AAR) in TTC-stained cardiac sections of the left ventricle were determined as previously described [32]. Briefly, Pale regions were regarded as the areas of necrosis $(\mathrm{AON})$. AON and AAR were calculated as the average percent area per slice from both sides of each section. Then, they were normalized to slice weight as follows: weight of total $\mathrm{AAR}=$ (weight of slice $1 \times \% \mathrm{AAR}$ of slice 1$)+($ weight of slice $2 \times \%$ AAR of slice 2$)+($ weight of slice $3 \times \%$ AAR of slice 3 ) + (weight of slice $4 \times \%$ AAR of slice 4$)+($ weight of slice $5 \times \%$ AAR of slice 5$)$. AON weight was calculated in the same manner.

Finally, infarct size was expressed as the percentage of the weight of AON to the weight of AAR [33]. If the AAR was $>90 \%$, this animal was excluded.

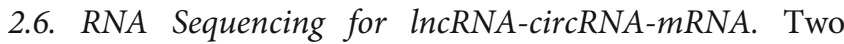
hours after reperfusion, the animals were quickly sacrificed to limit their suffering. The upper thoracic (T1-T4) spinal cord segments were immediately cut and frozen with liquid nitrogen and sent to Oebiotech Corporation (Shanghai, China) for RNA sequencing. The extraction of total RNA from T1-T4 spinal tissues was conducted by using the mirVana $^{\mathrm{TM}}$ PARIS ${ }^{\mathrm{TM}}$ Kit (Ambion-1556, USA) in accordance with the user manual. We assessed total RNA integrity using Agilent Bioanalyzer 2100 (Agilent Technologies). The spinal samples from the two groups were selected for microarray analysis. We used the Affymetrix ${ }^{\circledR}$ GeneChip ${ }^{\circledR}$ Whole Transcript Expression Arrays to analyze lncRNA and mRNA expression profiles, and we applied Agilent circRNA Microarray $8 \times 60 \mathrm{~K}$ to analyze circRNA expression profiles in the T1-T4 spinal cord segments [34]. Microarray data were obtained and analyzed by Oebiotech Corporation (Shanghai, China). The RNA-sequencing results were used to prioritize the heart-related spinal genes.

2.7. Identifying Differentially Expressed Genes and Gene Ontology (GO) Analysis. The differentially expressed mRNAs, lncRNAs, and circRNAs from the RNA-seq data were identified by using the edgeR algorithm. The mRNAs, lncRNAs, and circRNAs were deemed differentially expressed if they showed a false-discovery rate $(\mathrm{FDR})<5 \%$ and fold change $(\mathrm{FC})>2$. The molecular functions, cellular components, and biological processes of differentially expressed lncRNAs and circRNAs were described by using GO analysis (http://www.geneontology.org).

2.8. Kyoto Encyclopedia of Genes and Genomes (KEGG) Pathway Analysis. The gene set scores were calculated by using the FAIME algorithm based on the rank-weighted gene expression levels of individual samples (from the T1-T4 segments of the spinal cord), which converts each sample's transcriptomic profile to molecular mechanisms. KEGG analysis was applied to determine the biologic pathway roles of these differentially expressed lncRNAs and circRNAs based on the latest KEGG data (http://www.genome .jp/kegg/). Student's $t$ test was used to identify the differentially expressed KEGG pathways between IR and SD-IR samples. The KEGG pathways with adjusted $\mathrm{p}<0.05$ by Benjamini-Hochberg procedure were considered differentially expressed.

2.9. RT-qPCR Analysis for the Upper Thoracic Spinal Cord. The total RNA extracted from the T1-T4 segments of the spinal cord using the TRIzol ${ }^{\circledR}$ reagent (Invitrogen; Thermo Fisher Scientific, Inc.) in line with the manufacturer's instructions was used for the generation of cDNA. The primers were designed using the Primer Express 3.0 software (Applied Biosystems; Thermo Fisher Scientific, 


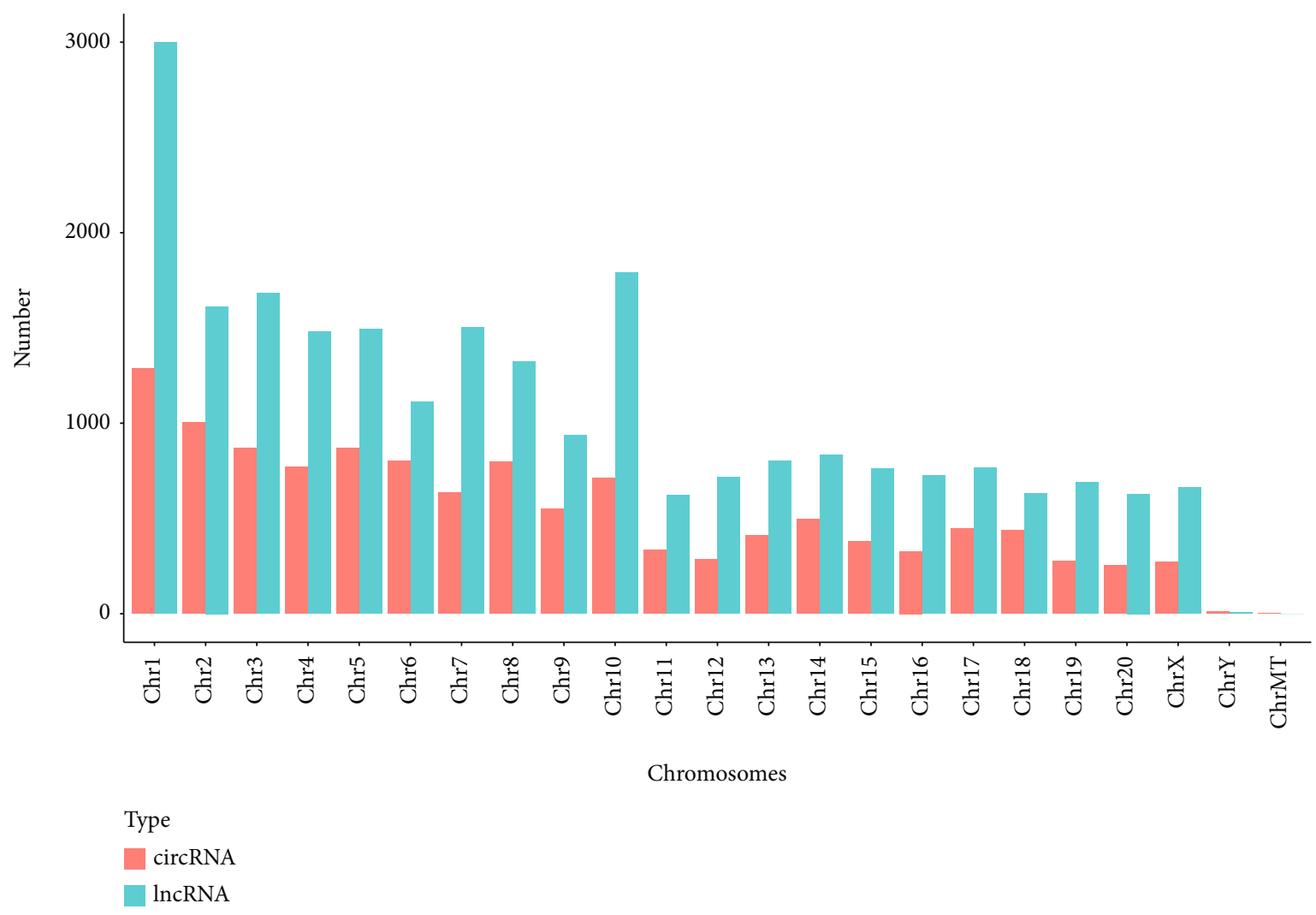

(a)

FIgUre 3: Continued. 


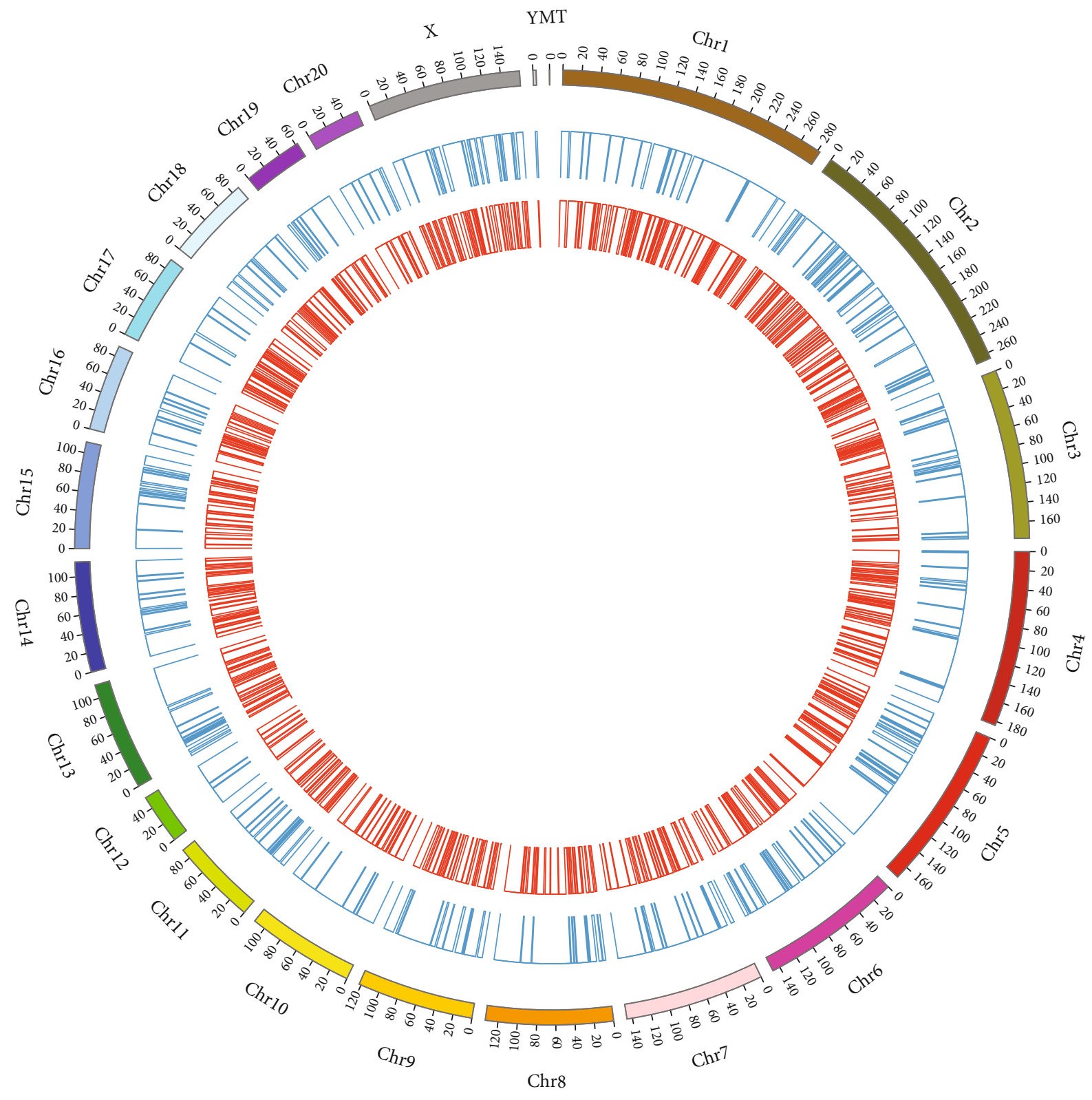

(b)

Figure 3: Continued. 

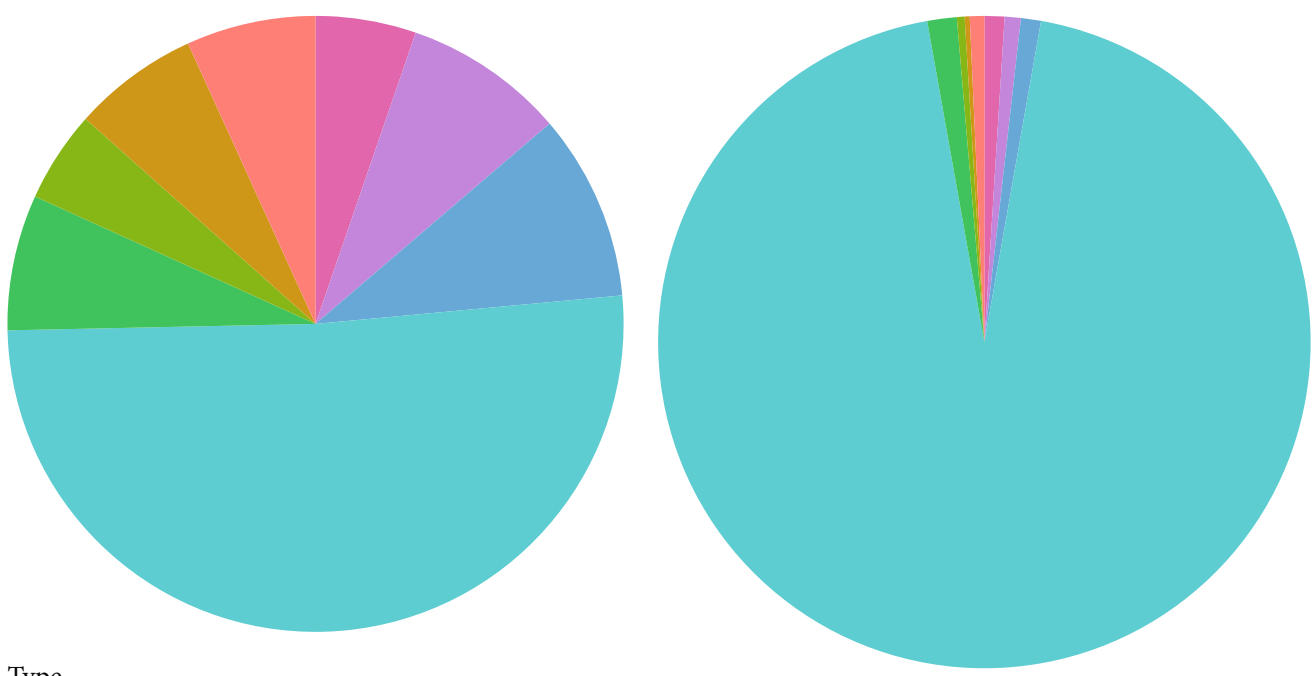

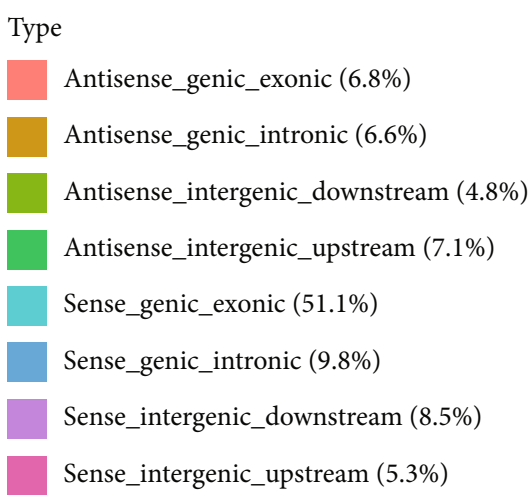

(c)

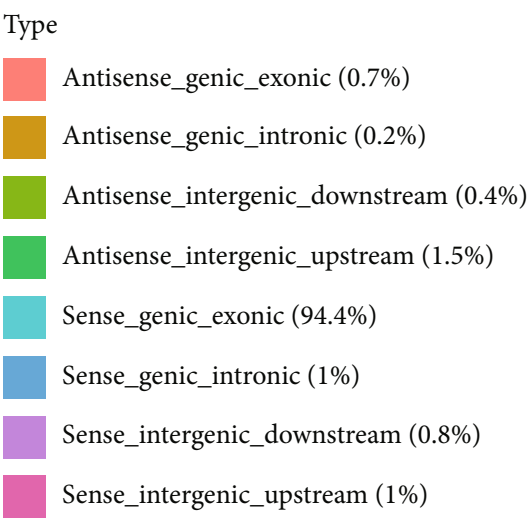

(d)

FIgURE 3: Comparison of the characteristics of lncRNAs, circRNAs, and mRNAs expression profiles in IR group and SD-IR group. (a) The distribution of lncRNAs and circRNAs on chromosomes. (b) LncRNAs and mRNAs on chromosomes. (c, d) Classification of lncRNAs and circRNAs.

Inc.); the specific forward and reverse primer sequences are listed in Table 1. RNA samples were quantified using a spectrophotometer (BioPhotometer; Eppendorf AG) and then synthesized to cDNA by reverse transcription using the PrimeScript ${ }^{\mathrm{TM}} \mathrm{RT}$ reagent kit (Takara Bio, Inc.). The temperature protocol was conducted using the following protocol: 15 minutes at $37^{\circ} \mathrm{C}, 5$ seconds at $85^{\circ} \mathrm{C}$, and held at $4^{\circ} \mathrm{C}$. cDNA was quantitated via RT-qPCR using a TB green ${ }^{\circledR}$ Premix Ex Taq (cat. no. RR420A; Takara Bio, Inc.). The thermocycling conditions for PCR were as follows: Initial denaturation for 30 seconds at $95^{\circ} \mathrm{C}$, followed by 40 cycles of 15 seconds at $95^{\circ} \mathrm{C}, 15$ seconds at $60^{\circ} \mathrm{C}$, and 45 seconds at $72^{\circ} \mathrm{C}$. The threshold cycle $(\mathrm{Cq})$ was used to estimate the amount of target mRNA. The comparative $\mathrm{Cq}$ method with a formula for relative FC $\left(2^{-\Delta \Delta \mathrm{Cq}}\right)$ was used to quantify the amplified transcripts. The relative gene expression levels were determined via normalization to GAPDH. Experiments were evaluated in triplicate and repeated $\geq 3$ times.

2.10. Construction of $\operatorname{lncRNA/circRNA-miRNA-mRNA}$ Network. The TargetScan (Release 7.2) and Miranda (version 0.10 .80 ) software were used to predict the relationship among lncRNA/circRNA, miRNAs, and mRNAs through base pairing. These predicted results were integrated to build the potential IncRNA/circRNA-miRNA-mRNA network. The Cytoscape software (version 3.7.2) was used to visualize the above data so as to explore the role of lncRNA/circRNAmiRNA-mRNA ceRNA network in the pathogenesis of MIRI after cardiac sympathetic denervation.

2.11. Computational Prediction of Protein-Protein Interaction (PPI) Analysis. The STRING database (ver. 10.5; https://string-db.org/) is an online database tool for searching known or predicted information on PPIs. The minimum PPI interaction score was set at 0.900 (highest confidence), and the wide disconnected node in the network was observed to obtain a complex PPI network of differentially expressed mRNAs. The Cytoscape software (version 3.7.2) was used to visualize the PPI network, and Cytohubba (a plug-in of Cytoscape) was used to identify the most relevant nodes by setting the degree. The PPI analysis was limited to an interaction threshold of 0.4 (medium confidence).

2.12. Statistical Analysis. Data were presented as the mean \pm SEM and were analyzed using GraphPad Prism software v5.0 (GraphPad Software, Inc.). Based on Gene Ontology 

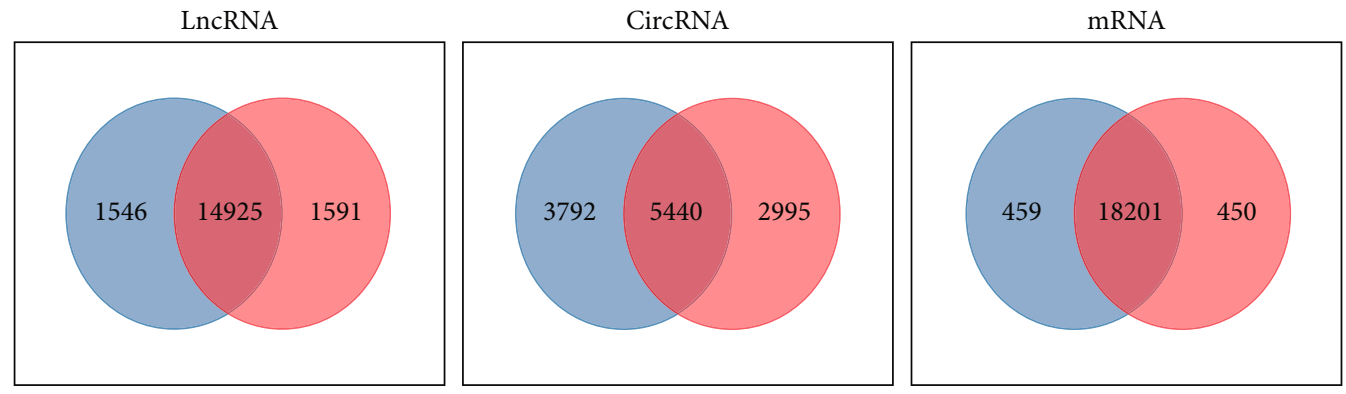

IR

SD_IR

(a)

SD_IR-vs-IR : pvalue $<0.05 \& \&|\log 2 \mathrm{FC}|>1$

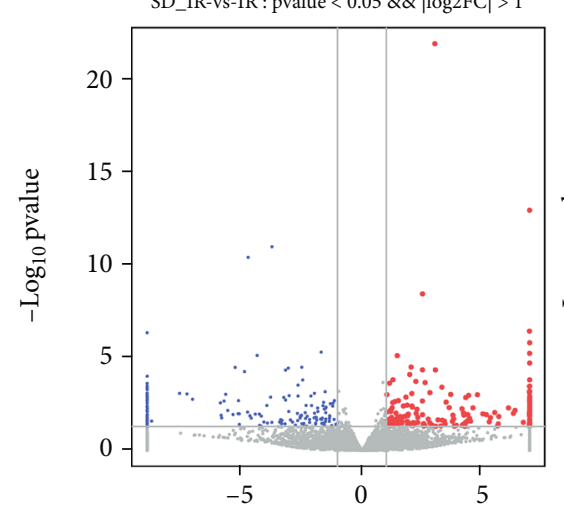

$\log _{2}$ fold change

SD_IR-vs-IR : pvalue $<0.05 \& \&|\log 2 \mathrm{FC}|>1$
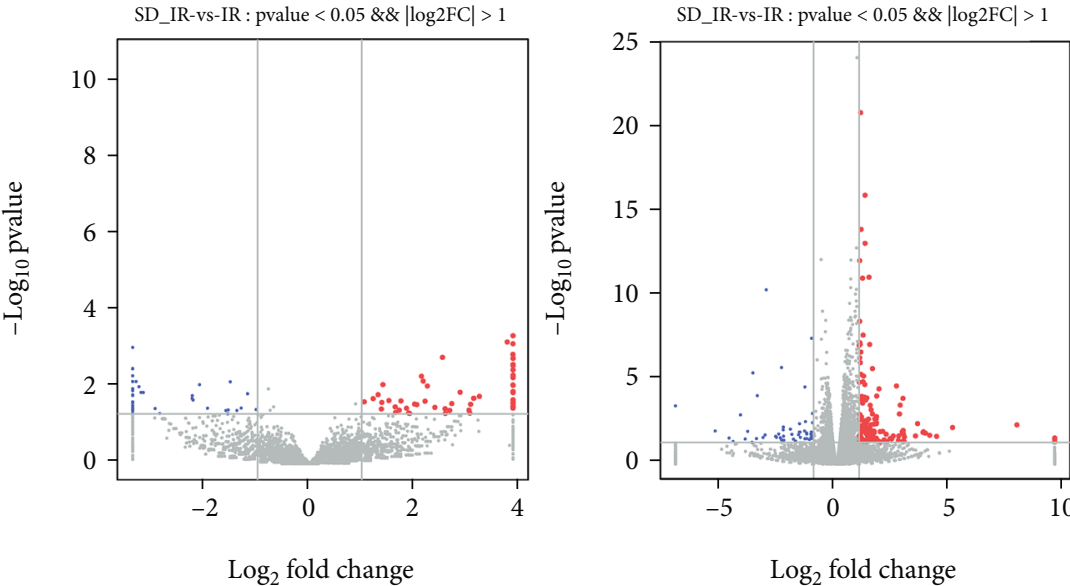

- Filtered

- Up

- Down

(b)

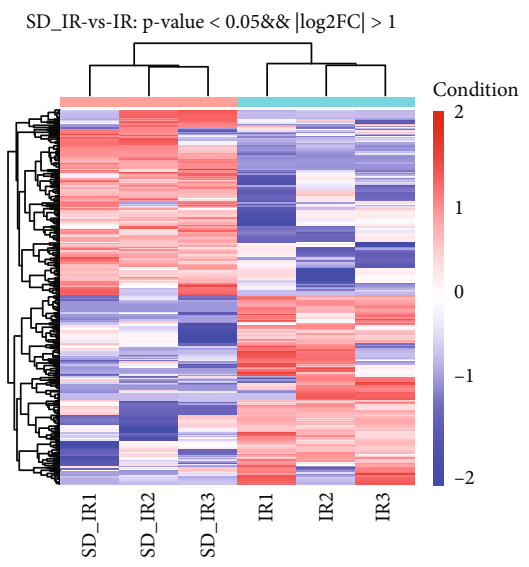

SD_IR-vs-IR: p-value $<0.05 \& \&|\log 2 \mathrm{FC}|>1$

SD_IR-vs-IR: p-value $<0.05 \& \&|\log 2 \mathrm{FC}|>1$
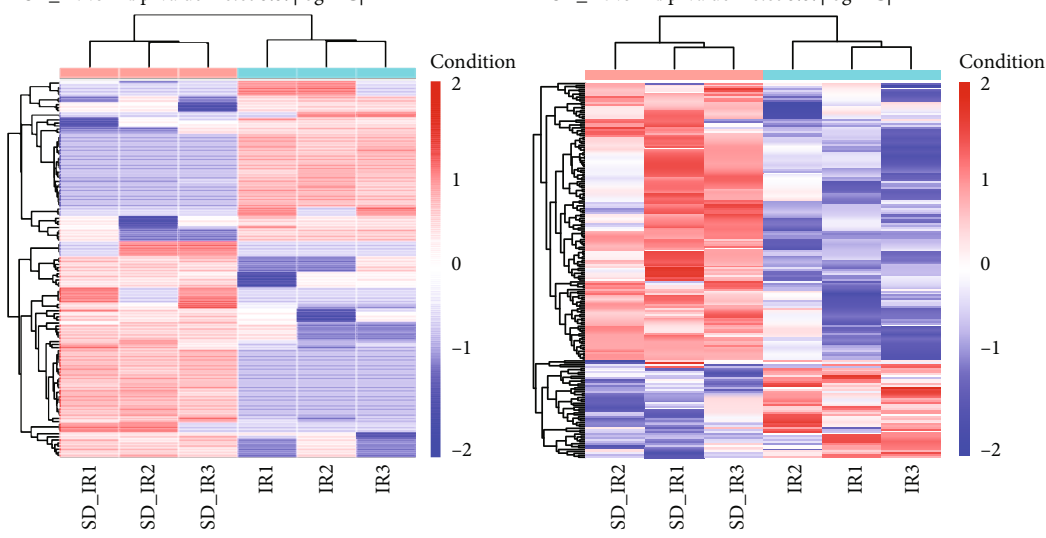

Condition

IR

SD_IR

(c)

Figure 4: Differential expression patterns of lncRNAs, circRNAs, and mRNAs. (a) The specific lncRNAs, circRNAs, and mRNAs shared between IR group and SD-IR group. (b) The volcano plot of lncRNAs, circRNAs, and mRNAs expression. Red color is indicative of upregulated and blue color is indicative of downregulated genes, where $\mathrm{p}<0.05$ and $|\mathrm{FC}|>2$ are considered statistically significant; grey color is indicative of nonsignificantly different genes. (c) The hierarchical heat map shows the deregulated lncRNAs, circRNAs, and mRNAs in the T1-T4 spinal cord segments between IR group and SD-IR group; up- and downregulated genes are colored in red and green, respectively $\left(\mathrm{p}<0.05\right.$ and $\left.\log _{2}|\mathrm{FC}|>1\right)$. 
TABLE 2: The detail information of the top 30 up-regulated mRNAs in the T1-4 spinal cord between SD-IR group and IR group.

\begin{tabular}{|c|c|c|c|}
\hline Gene ID & $\log _{2} \mathrm{FC}(\mathrm{SD}-\mathrm{IR} / \mathrm{IR})$ & Pvalue & Description \\
\hline LOC102553010 & 4.40 & 0.021887 & Leukocyte elastase inhibitor A-like \\
\hline RGD1359290 & 4.10 & 0.019123 & Ribosomal_L22 domain containing protein RGD1359290 \\
\hline LOC108348083 & 3.91 & 0.014045 & Delta-1-pyrroline-5-carboxylate synthase \\
\hline RGD1305184 & 3.83 & 0.011827 & Similar to CDNA sequence $\mathrm{BC} 023105$ \\
\hline Gns & 3.81 & 0.012511 & Glucosamine (N-acetyl)-6-sulfatase \\
\hline Prf1 & 3.57 & 0.003838 & Perforin 1 \\
\hline LOC100911034 & 3.47 & 0.020641 & Cysteine desulfurase, mitochondrial-like \\
\hline Ubd & 2.99 & 0.024065 & Ubiquitin $\mathrm{D}$ \\
\hline Mcpt9 & 2.96 & 0.016414 & Mast cell protease 9 \\
\hline LOC100910446 & 2.95 & 0.039393 & Syntaxin-7-like \\
\hline Sctr & 2.94 & 0.009767 & Secretin receptor \\
\hline Ccl12 & 2.92 & 0.000115 & Chemokine (C-C motif) ligand 12 \\
\hline Cxcl10 & 2.89 & 0.011969 & $\mathrm{C}-\mathrm{X}-\mathrm{C}$ motif chemokine ligand 10 \\
\hline Ngp & 2.81 & 0.000296 & Neutrophilic granule protein \\
\hline Rhag & 2.78 & 0.001015 & Rh-associated glycoprotein \\
\hline Cxcl11 & 2.74 & 0.020485 & C-X-C motif chemokine ligand 11 \\
\hline Car1 & 2.64 & $2.06 \mathrm{E}-05$ & Carbonic anhydrase I \\
\hline Padi3 & 2.50 & 0.02082 & Peptidyl arginine deiminase 3 \\
\hline S100a5 & 2.43 & 0.031729 & S100 calcium binding protein A5 \\
\hline Unc45b & 2.19 & 0.016491 & Unc-45 myosin chaperone B \\
\hline $\mathrm{Cd} 5 \mathrm{l}$ & 2.10 & 0.034827 & Cd5 molecule-like \\
\hline Ermap & 2.03 & 0.010957 & Erythroblast membrane-associated protein \\
\hline Capn13 & 1.94 & 0.046362 & Calpain 13 \\
\hline Clec5a & 1.87 & $3.08 \mathrm{E}-05$ & C-type lectin domain family 5 , member A \\
\hline Gbp1 & 1.84 & 0.040079 & Guanylate binding protein 1 \\
\hline Hemgn & 1.77 & $8.40 \mathrm{E}-05$ & Hemogen \\
\hline Cxcl13 & 1.76 & 0.00143 & C-X-C motif chemokine ligand 13 \\
\hline Kel & 1.76 & 0.00334 & Kell blood group, metallo-endopeptidase \\
\hline LOC680322 & 1.75 & 0.028182 & Similar to histone $\mathrm{H} 2 \mathrm{~A}$ type 1 \\
\hline Ms4a3 & 1.75 & 0.003821 & Membrane spanning 4-domains A3 \\
\hline
\end{tabular}

$|\log 2 \mathrm{FC}|>1 ; \mathrm{p}<0.05$ by analysis of variance.

Biological Process (GOBP) definition, Fisher's exact test was applied to determine whether the proportion of differentially expressed genes in a given GOBP gene set was significantly enhanced. RT-qPCR parameters were analyzed using the unpaired $t$ test for repeated measures, with $P$ value less than 0.05 was considered statistically significant.

\section{Results}

3.1. Chemical Sympathectomy-Induced Cardiac Alterations. The level of NE in the cardiac tissues of the SD-IR group $(\mathrm{n}=6,0.1650 \pm 0.1057 \mathrm{ng} / \mathrm{mg})$ was significantly lower compared with that in the IR group $(n=6,2.687 \pm 0.1349 \mathrm{ng} / \mathrm{mg})$ (Figure 1(a)).

3.2. Characteristics of Myocardial Ischemic Tissue. In the two groups, we observed the development of ST-segment elevation and QRS complex changes on an electrocardiogram; moreover, there was a cyanotic change in the myocardium of the occluded area 30 minutes after cardiac ischemia. Serum cardiac troponin cTnI $(0.73 \pm 0.26 \mu \mathrm{g} / \mathrm{L})$ in the SDIR group was significantly lower than that in the IR group $(15.14 \pm 2.44 \mu \mathrm{g} / \mathrm{L}) 2$ hours after reperfusion (Figure 1(b)). These results verified the successful occlusion of the LAD.

In the IR group, a structural disorder of the cardiac tissue was observed, with different degrees of vacuolar degeneration and necrosis, as well as loose stroma (Figure 1(c)). Moreover, the number of cardiomyocyte fibers was markedly increased after IR. In the SD-IR group, myocardium showed a better architecture, and the myocardial fibers and myocardial cells were relatively intact and arranged in an orderly manner (Figure $1(\mathrm{c})$ ).

The results from TTC staining clearly exhibited a reduced myocardial infarction-indicated by the pale color region in the transverse section of heart-in the IR group (Figure 1(d)). To ensure that the difference in the infarct size was not caused by different myocardium injuries, we measured the area at risk (AAR) and found no difference 
TABLE 3: The detail information of the top 30 down-regulated mRNAs in the T1-4 spinal cord between SD-IR group and IR group.

\begin{tabular}{|c|c|c|c|}
\hline Gene ID & $\begin{array}{c}\log _{2} \mathrm{FC} \\
(\mathrm{SD}-\mathrm{IR} / \mathrm{IR})\end{array}$ & Pvalue & Description \\
\hline LOC100912599 & -5.31 & 0.010395 & NADH dehydrogenase [ubiquinone] iron-sulfur protein 6 , mitochondrial-like \\
\hline LOC100911994 & -3.89 & 0.01095 & Coiled-coil domain-containing protein 132-like \\
\hline Clcn2 & -3.51 & 0.029527 & Chloride channel, voltage-sensitive 2 \\
\hline LOC100910308 & -3.47 & 7.97E-05 & Multifunctional protein ADE2-like \\
\hline LOC100910207 & -3.12 & 0.018014 & Protein Dr1-like \\
\hline Vwa5a & -2.62 & 0.02532 & von Willebrand factor A domain containing $5 \mathrm{~A}$ \\
\hline Dpep1 & -2.53 & 0.015698 & Dipeptidase 1 (renal) \\
\hline NEWGENE_1582994 & -2.45 & 0.02675 & DCN1, defective in cullin neddylation 1 , domain containing 2 \\
\hline LOC103689986 & -2.41 & $1.64 \mathrm{E}-06$ & Protein YIF1B \\
\hline Iqcf3 & -2.40 & 0.036362 & IQ motif containing F3 \\
\hline Ahsg & -2.32 & 0.021173 & Alpha-2-HS-glycoprotein \\
\hline Hpgd & -2.01 & 0.008083 & Hydroxyprostaglandin dehydrogenase 15 (NAD) \\
\hline RGD1561778 & -1.94 & 0.016898 & Similar to dendritic cell-derived immunoglobulin(Ig)-like receptor 1, DIgR1 - mouse \\
\hline LOC297568 & -1.88 & 0.032025 & Alpha-1-inhibitor III \\
\hline Rlim & -1.78 & 0.042544 & Ring finger protein, LIM domain interacting \\
\hline Slc39a12 & -1.65 & 0.017633 & Solute carrier family 39 member 12 \\
\hline LOC100911865 & -1.62 & $1.20 \mathrm{E}-02$ & TBC1 domain family member 12 -like \\
\hline Myo18b & -1.61 & 0.003815 & Myosin XVIIIb \\
\hline Tspan10 & -1.59 & 0.012324 & Tetraspanin 10 \\
\hline Sds & -1.57 & 0.025502 & Serine dehydratase \\
\hline Trim63 & -1.39 & 0.010581 & Tripartite motif containing 63 \\
\hline LOC102550588 & -1.38 & 0.030436 & Zinc finger protein 709-like \\
\hline Sh2d $4 b$ & -1.37 & $2.44 \mathrm{E}-05$ & SH2 domain containing $4 \mathrm{~B}$ \\
\hline Hif3a & -1.35 & 0.002856 & Hypoxia inducible factor 3 , alpha subunit \\
\hline Galnt15 & -1.30 & 0.01374 & Polypeptide N-acetylgalactosaminyltransferase 15 \\
\hline LOC687780 & -1.30 & 0.029663 & Similar to Finkel-Biskis-Reilly murine sarcoma virusubiquitously expressed \\
\hline Trpv4 & -1.29 & 0.015774 & Transient receptor potential cation channel, subfamily V, member 4 \\
\hline Mt1 & -1.18 & 0.033903 & Metallothionein 1 \\
\hline Fbxw10 & -1.11 & 0.016073 & F-box and WD repeat domain containing 10 \\
\hline Dcst1 & -1.10 & 0.024549 & DC-STAMP domain containing 1 \\
\hline
\end{tabular}

$|\log 2 \mathrm{FC}|>1 ; \mathrm{p}<0.05$ by analysis of variance.

between the two groups (Figure 1(e)). TTC staining showed a significant reduction in infarct size in the SD-IR group $(9.26 \pm 0.16 \%, \mathrm{n}=6)$ compared with the IR group (15.05 $\pm 0.70 \%, \mathrm{n}=6)$ (Figure 1(e)).

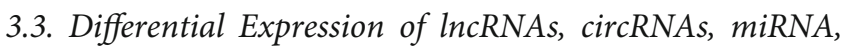
and $m R N A s$. To fully understand the role of cardiac sympathetic denervation in myocardial ischemia/reperfusion injury, we simultaneously analyzed the profiles of differential expression of lncRNAs, circRNAs, miRNAs, and mRNAs through microarray analysis. Significant difference was defined as fold change $\geq 2$ and $p<0.05$. In this study, the SD-IR group identified 333 lncRNAs, 122 circRNAs, 23 miRNAs, and 199 mRNAs with significant differential expression (Figure 2). Through high-throughput RNA sequencing, we found that $165 \operatorname{lncRNAs}$ were upregulated and 168 lncRNAs were downregulated; 70 circRNAs were upregulated and 52 circRNAs were downregulated; $12 \mathrm{miR}$ NAs were upregulated and 11 miRNAs were downregulated; and 148 mRNAs were upregulated and 51 mRNAs were downregulated (Figure 2).

A total of 23711 lncRNAs and 12007 circRNAs (Figures 3(a) and 3(b)) were identified in all chromosomes. Among lncRNAs, most (51.1\%) were sense_genic_exonic lncRNA, followed by sense_genic_intronic lncRNAs (9.8\%), sense_intergenic_downstream lncRNAs (8.5\%), sense_intergenic_upstream lncRNAs (5.3\%), antisense_genic_exonic lncRNA (6.8\%), antisense_genic_intronic lncRNAs (6.6\%), antisense_intergenic_downstream lncRNAs (4.8\%), and antisense_intergenic_upstream lncRNAs (7.1\%) (Figure 3(c)). In circRNA, the vast majority (94.4\%) were sense_genic_exonic circRNA, followed by sense_genic_intronic circRNAs (1\%), sense_intergenic_downstream circRNAs (0.8\%), sense_intergenic_upstream circRNAs (1\%), antisense_genic_exonic 
TABLE 4: The detail information of the top 30 up-regulated lncRNAs and top 30 down-regulated lncRNAs in the T1-4 spinal cord between SD-IR group and IR group.

\begin{tabular}{|c|c|c|c|c|c|}
\hline \multicolumn{3}{|c|}{ Upregulation } & \multicolumn{3}{|c|}{ Downregulation } \\
\hline LncRNA ID & $\log _{2} \mathrm{FC}(\mathrm{SD}-\mathrm{IR} / \mathrm{IR})$ & Pvalue & LncRNA ID & $\log _{2} \mathrm{FC}(\mathrm{SD}-\mathrm{IR} / \mathrm{IR})$ & Pvalue \\
\hline NONRATT015643.2 & 6.61 & 0.030213 & NONRATT002082.2 & -8.80 & 0.003863 \\
\hline NONRATT003628.2 & 6.28 & 0.006736 & NONRATT004821.2 & -8.62 & 0.025764 \\
\hline NONRATT005973.2 & 6.21 & 0.009866 & NONRATT010722.2 & -7.18 & 0.000854 \\
\hline NONRATT016279.2 & 6.00 & 0.004952 & NONRATT000247.2 & -5.80 & 0.00261 \\
\hline NONRATT008379.2 & 5.61 & 0.014618 & NONRATT027814.2 & -5.77 & 0.012322 \\
\hline NONRATT013717.2 & 5.58 & 0.036523 & NONRATT008629.2 & -5.75 & 0.017423 \\
\hline NONRATT016674.2 & 5.56 & 0.047236 & NONRATT005321.2 & -5.65 & 0.002079 \\
\hline NONRATT017458.2 & 5.44 & 0.008762 & NONRATT028463.2 & -5.59 & 0.000887 \\
\hline NONRATT002859.2 & 5.27 & 0.016794 & TCONS_00021543 & -5.51 & 0.006752 \\
\hline NONRATT009760.2 & 5.22 & 0.028265 & NONRATT004098.2 & -5.28 & 0.011345 \\
\hline TCONS_00002734 & 5.09 & 0.011358 & NONRATT007713.2 & -5.20 & $3.10 \mathrm{E}-05$ \\
\hline NONRATT011768.2 & 4.95 & 0.01038 & NONRATT004090.2 & -5.07 & 0.010222 \\
\hline NONRATT009811.2 & 4.73 & 0.000946 & NONRATT018759.2 & -5.06 & 0.001982 \\
\hline NONRATT026200.2 & 4.54 & 0.004819 & NONRATT003447.2 & -5.03 & 0.010344 \\
\hline NONRATT030638.2 & 4.44 & 0.025926 & NONRATT007815.2 & -5.02 & 0.039319 \\
\hline NONRATT008615.2 & 4.37 & 0.001033 & NONRATT025664.2 & -4.82 & $5.13 \mathrm{E}-05$ \\
\hline NONRATT012797.2 & 4.35 & 0.023183 & NONRATT019695.2 & -4.69 & 0.008267 \\
\hline NONRATT020189.2 & 4.33 & 0.011361 & NONRATT006541.2 & -4.66 & $3.19 \mathrm{E}-11$ \\
\hline NONRATT020809.2 & 4.27 & 0.02875 & NONRATT019538.2 & -4.60 & 0.013069 \\
\hline NONRATT029190.2 & 4.25 & 0.001325 & NONRATT026641.2 & -4.54 & 0.01747 \\
\hline NONRATT022867.2 & 4.24 & 0.031209 & NONRATT024737.2 & -4.38 & 0.010641 \\
\hline NONRATT000517.2 & 4.24 & 0.031431 & TCONS_00013588 & -4.30 & $6.80 \mathrm{E}-06$ \\
\hline NONRATT003681.2 & 4.18 & 0.012182 & NONRATT020675.2 & -4.21 & 0.010623 \\
\hline NONRATT021791.2 & 4.16 & 0.009107 & NONRATT010709.2 & -4.20 & 0.043975 \\
\hline NONRATT023302.2 & 4.14 & 0.038514 & NONRATT022516.2 & -4.14 & 0.048093 \\
\hline NONRATT003838.2 & 4.12 & 0.043331 & NONRATT020315.2 & -4.13 & 0.01375 \\
\hline NONRATT018320.2 & 4.10 & 0.005454 & TCONS_00002731 & -4.02 & 0.028687 \\
\hline NONRATT013257.2 & 3.87 & 0.043965 & NONRATT006149.2 & -3.89 & 0.003669 \\
\hline NONRATT000472.2 & 3.79 & 0.048619 & NONRATT011296.2 & -3.87 & 0.033214 \\
\hline NONRATT005188.2 & 3.76 & 0.019322 & TCONS_00007997 & -3.75 & 0.001282 \\
\hline
\end{tabular}

$|\log 2 \mathrm{FC}|>1 ; \mathrm{p}<0.05$ by analysis of variance.

circRNA (0.7\%), antisense_genic_intronic circRNAs (0.2\%), antisense_intergenic_downstream circRNAs (0.4\%), and antisense_intergenic_upstream circRNAs (1.5\%) (Figure 3(d)).

3.4. Differential Expression Patterns of mRNAs, IncRNAs, and circRNAs in MIRI. The expression patterns of mRNAs, lncRNAs, and circRNAs in the T1-T4 spinal cord 2 hours after MIRI were examined using microarray. From the volcano map and hierarchical clustering analysis results between the SD-IR group and the IR group, a landscape of the expression characteristics of the mRNAs, IncRNAs, and circRNAs was obtained (Figure 4(a)). The volcano plots demonstrated that large numbers of mRNAs, lncRNAs, and circRNAs were differentially expressed between the two groups (Figure 4(b)). Furthermore, these differential alterations of mRNA, IncRNA, and circRNA expression in the T1-T4 spinal cord were associated with cardiac sympathetic denervation.
The hierarchical heat map showed the deregulated mRNAs, lncRNAs, and circRNAs in the T1-T4 spinal cord segments between the SD-IR group and the IR group (Figure 4(c)); up- and downregulated genes are colored in red and green, respectively $(\mathrm{p}<0.05$ and $\log 2|\mathrm{FC}|>1)$.

3.5. Analysis of mRNAs, IncRNAs, circRNAs, and miRNAs Changes in the Spinal Cord after Cardiac Sympathetic Denervation. Among the differentially expressed mRNAs, there were 199 genes exhibiting fold change (FC) higher than 1. The number of downregulated mRNAs was 51 , whereas the number of upregulated mRNAs was 148 . The most upregulated mRNAs were Fxyd2, Tyrp1, Il31ra, RT1-CE4, Scn11a, MGC108823, Tnc, Irf8, and MGC105567. The most downregulated mRNAs were LOC100911256, Cyp4b1, LOC103689986, LOC103693165, Sh2d4b, LOC100910308, Nfs1, Prex2, LOC103691806, and Hif3a. The detailed 
TABLE 5: The detail information of the top 20 up-regulated circRNAs in the T1-4 spinal cord between SD-IR group and IR group.

\begin{tabular}{|c|c|c|}
\hline CircRNA ID & $\log _{2} \mathrm{FC}(\mathrm{SD}-\mathrm{IR} / \mathrm{IR})$ & Pvalue \\
\hline circRNA_06761|Chr3:13146571_13147147_- & 3.91 & 0.000446 \\
\hline circRNA_03748|Chr15:51936366_51954838_- & 3.80 & 0.000648 \\
\hline circRNA_01962|Chr10:103586432_103677527_- & 3.27 & 0.017323 \\
\hline circRNA_11924|Chr9:119105074_119127222_+ & 3.16 & 0.019308 \\
\hline circRNA_01988|Chr10:109580083_109583063_- & 3.16 & 0.01944 \\
\hline circRNA_04213|Chr16:81879800_81897683_- & 3.10 & 0.027871 \\
\hline circRNA_05436|Chr2:5570780_5576549_- & 3.08 & 0.047573 \\
\hline circRNA_09388|Chr6:38617368_38619590_+ & 3.06 & 0.03965 \\
\hline circRNA_04650|Chr17:86845723_86873000_- & 2.89 & 0.013496 \\
\hline circRNA_02217|Chr11:70539679_70570814_- & 2.74 & 0.026695 \\
\hline circRNA_06237|Chr2:218919131_218929338_- & 2.69 & 0.040249 \\
\hline circRNA_11356|Chr8:128384378_128391077_- & 2.61 & 0.04823 \\
\hline circRNA_08473|Chr5:50318351_50319000_- & 2.60 & 0.036427 \\
\hline circRNA_02159|Chr11:47165426_47169764_+ & 2.56 & 0.001643 \\
\hline circRNA_05629|Chr2:45801786_45859187_+ & 2.41 & 0.033552 \\
\hline circRNA_09485|Chr6:60609626_60633969_+ & 2.26 & 0.009238 \\
\hline circRNA_06869|Chr3:33465220_33473531_+ & 2.22 & 0.023101 \\
\hline circRNA_00547|Chr1:143712491_143723739_- & 2.19 & 0.006884 \\
\hline circRNA_08772|Chr5:123824724_123825786_+ & 2.15 & 0.005133 \\
\hline circRNA_01552|Chr10:55283300_55338553_+ & 2.06 & 0.028481 \\
\hline
\end{tabular}

$|\log 2 \mathrm{FC}|>1 ; \mathrm{p}<0.05$ by analysis of variance.

TABLE 6: The detail information of the top 20 down-regulated circRNAs in the T1-4 spinal cord between SD-IR group and IR group.

\begin{tabular}{|c|c|c|}
\hline CircRNA ID & $\log _{2} \mathrm{FC}(\mathrm{SD}-\mathrm{IR} / \mathrm{IR})$ & Pvalue \\
\hline circRNA_06863|Chr3:29584278_29597359_- & -3.41 & 0.004976 \\
\hline circRNA_11934|Chr9:119750465_119765191_- & -3.34 & 0.007029 \\
\hline circRNA_02911|Chr13:92762599_92783433_+ & -3.28 & 0.009623 \\
\hline circRNA_04274|Chr17:5524117_5571375_+ & -3.25 & 0.013595 \\
\hline circRNA_04457|Chr17:53489461_53499989_+ & -3.20 & 0.013695 \\
\hline circRNA_11984|ChrX:13905156_13917538_+ & -2.97 & 0.035854 \\
\hline circRNA_08837|Chr5:129413066_129491380_+ & -2.89 & 0.048702 \\
\hline circRNA_03573|Chr15:13454469_13468631_- & -2.26 & 0.019466 \\
\hline circRNA_07224|Chr3:113195876_113197193_- & -2.26 & 0.016518 \\
\hline circRNA_07819|Chr4:64451679_64474930_- & -2.24 & 0.021549 \\
\hline circRNA_04050|Chr16:23555919_23573530_+ & -2.12 & 0.00851 \\
\hline circRNA_09970|Chr7:9826042_9826424_- & -1.97 & 0.035185 \\
\hline circRNA_11137|Chr8:96067918_96073130_- & -1.61 & 0.040373 \\
\hline circRNA_07862|Chr4:66193731_66214585_+ & -1.57 & 0.049364 \\
\hline circRNA_07510|Chr3:175512077_175535023_+ & -1.57 & 0.038954 \\
\hline circRNA_11420|Chr9:8350080_8421978_+ & -1.53 & 0.007153 \\
\hline circRNA_03499|Chr14:113838631_113857380_+ & -1.40 & 0.04037 \\
\hline circRNA_09492|Chr6:64852693_64861750_+ & -1.32 & 0.035057 \\
\hline circRNA_02339|Chr12:587083_591296_- & -1.19 & 0.014725 \\
\hline circRNA_04622|Chr17:84855705_84901793_+ & -1.03 & 0.038253 \\
\hline
\end{tabular}

$|\log 2 \mathrm{FC}|>1 ; \mathrm{p}<0.05$ by analysis of variance. 
TABLE 7: The detail information of differentially expressed miRNAs in the T1-4 spinal cord between SD-IR group and IR group.

\begin{tabular}{|c|c|c|c|}
\hline miRNA_ID & $\log _{2}$ FC (SD-IR/IR) & Pvalue & Length \\
\hline \multicolumn{4}{|l|}{ Upregulation } \\
\hline novel21_star & 3.695484 & 0.021677 & 23 \\
\hline Rno-miR-293-5p & 2.798924 & 0.000538 & 21 \\
\hline novel98_mature & 2.479701 & 0.03268 & 23 \\
\hline novel174_mature $>$ novel176_mature $>$ novel705_mature & 2.236875 & 0.046924 & 22 \\
\hline Rno-miR-146a-3p & 2.118125 & 0.026213 & 21 \\
\hline Rno-miR-96-5p & 0.903083 & 0.005817 & 23 \\
\hline Rno-miR-183-5p & 0.614791 & 0.003377 & 22 \\
\hline Rno-miR-493-5p & 0.60771 & 0.007994 & 22 \\
\hline Rno-miR-363-3p & 0.60157 & 0.019368 & 21 \\
\hline Rno-miR-3553 & 0.600458 & 0.03527 & 23 \\
\hline \multicolumn{4}{|l|}{ Downregulation } \\
\hline novel190_mature & -3.63316 & 0.016898 & 23 \\
\hline novel275_mature>novel301_mature & -3.4838 & 0.045199 & 24 \\
\hline novel586_mature & -3.41968 & 0.000414 & 23 \\
\hline novel88_mature & -2.24793 & 0.005616 & 23 \\
\hline novel252_mature & -1.81632 & 0.044256 & 23 \\
\hline novel62_mature & -1.46827 & 0.03968 & 24 \\
\hline Rno-miR-206-3p & -1.07274 & 0.014845 & 22 \\
\hline Rno-miR-1-3p & -0.86313 & 0.029666 & 22 \\
\hline Rno-miR-7a-2-3p & -0.67697 & 0.040237 & 22 \\
\hline
\end{tabular}

$|\log 2 \mathrm{FC}|>1 ; \mathrm{p}<0.05$ by analysis of variance.

information regarding the differentially expressed mRNAs is listed in Tables 2 and 3.

The results showed that 333 lncRNAs, including 165 upregulated and 168 downregulated lncRNAs, were significantly altered in the SD-IR group compared with the IR group. The most upregulated lncRNAs were NONRATT003225.2, TCONS_00000042, NONRATT013473.2, NONRATT011603.2, NONRATT018299.2, NONRATT001917.2, NONRATT017719.2, NONRATT004831.2, TCONS_00008547, and NONRATT025548.2. The most downregulated lncRNAs were NONRATT003165.2, NONRATT006541.2, NONRATT031746.1, TCONS_00013588, TCONS_00009293, NONRATT007713.2, NONRATT016595.2, NONRATT011191.2, NONRATT025664.2, and NONRATT010240.2. Additional information regarding the differentially expressed lncRNAs is presented in Table 4.

Compared with the IR group, the SD-IR group showed 70 upregulated and 52 downregulated circRNAs. The most upregulated circRNAs were circRNA_00336, circRNA_ 00446, circRNA_00547, circRNA_01552, circRNA_01962, circRNA_01988, circRNA_02159, circRNA_02217, circRNA_03207, and circRNA_03748. The most downregulated circRNAs were circRNA_02339, circRNA_02911, circRNA_03499, circRNA_03573, circRNA_04050, circRNA_04274, circRNA_04457, circRNA_04622, circRNA_ 06863, and circRNA_07224. Additional information regarding the differentially expressed circRNAs is presented in Tables 5 and 6 .

Among the differentially expressed miRNAs, there were 12 upregulated and 11 downregulated miRNAs in the SD-IR group compared with the IR group. The upregulated miRNAs were rno-miR-293-5p, rno-miR-183-5p, rno-miR-96-5p, rno-miR-493-5p, novel248_mature, rnomiR-363-3p, novel21_star, rno-miR-146a-3p, novel98_ mature, rno-miR-3553, novel438_mature, and novel174_ mature $>$ novel176_mature $>$ novel705_mature. The downregulated miRNAs were novel586_mature, novel88_mature, novel342_mature, rno-miR-206-3p, novel190_mature, rnomiR-1-3p, novel655_mature, novel62_mature, rno-miR-7a$2-3$ p, novel252_mature, and novel275_mature $>$ novel301_ mature. Additional information regarding the differentially expressed miRNAs is shown in Table 7.

3.6. GO and KEGG Analysis of Differentially Expressed $m R N A s$, $\ln c R N A s$, circRNAs, and miRNAs. To investigate the spinal molecular mechanisms of cardiac sympathetic denervation on MIRI, we performed GO and KEGG pathway analyses of the differentially expressed mRNAs (DEM), lncRNAs (DEL), circRNAs (DEC), and miRNAs in SD-MIRI vs. IR group (Tables 8 and 9).

Based on GO analysis, DEL focusing on cell components were related to the Golgi cisterna, membrane, axon, cytoplasm, and cytoplasmic microtubules, while those focusing on molecular function (MF) were related to translation initiation factor activity, protein tyrosine kinase binding, myosin binding, and SNAP receptor activity (Figure 5(a)). The GO function prediction showed that DEC focusing on cell components were related to the VCP-NPL4-UFD1 AAA ATPase complex, nuclear chromosome telomeric region, and actin cytoskeleton, whereas those focusing on molecular functions 
TABLe 8: The Gene Ontology (GO) terms enriched for the differentially expressed genes.

\begin{tabular}{|c|c|c|c|}
\hline GO ID & Term & Gene number & Pvalue \\
\hline \multicolumn{4}{|c|}{ biological_process } \\
\hline GO:0035458 & Cellular response to interferon-beta & 12 & $1.80 \mathrm{E}-18$ \\
\hline GO:0006952 & Defense response & 11 & $2.39 \mathrm{E}-14$ \\
\hline GO:0071346 & Cellular response to interferon-gamma & 9 & $2.22 \mathrm{E}-08$ \\
\hline GO:0071222 & Cellular response to lipopolysaccharide & 11 & $2.09 \mathrm{E}-07$ \\
\hline GO:0050832 & Defense response to fungus & 4 & $8.84 \mathrm{E}-07$ \\
\hline GO:0070098 & Chemokine-mediated signaling pathway & 6 & $9.17 \mathrm{E}-07$ \\
\hline GO:0031640 & Killing of cells of other organism & 4 & $2.35 \mathrm{E}-06$ \\
\hline GO:0032496 & Response to lipopolysaccharide & 12 & $2.41 \mathrm{E}-06$ \\
\hline GO:0042742 & Defense response to bacterium & 8 & $2.62 \mathrm{E}-06$ \\
\hline GO:0019731 & Antibacterial humoral response & 4 & $3.59 \mathrm{E}-06$ \\
\hline \multicolumn{4}{|c|}{ cellular_component } \\
\hline GO:0005615 & Extracellular space & 34 & $8.43 \mathrm{E}-09$ \\
\hline GO:0005623 & Cell & 6 & $4.24 \mathrm{E}-05$ \\
\hline GO:0030863 & Cortical cytoskeleton & 3 & 0.00016 \\
\hline GO:0031012 & Extracellular matrix & 8 & 0.000245 \\
\hline GO:0000786 & Nucleosome & 4 & 0.000876 \\
\hline GO:0009897 & External side of plasma membrane & 8 & 0.001475 \\
\hline GO:0005578 & Proteinaceous extracellular matrix & 7 & 0.001944 \\
\hline GO:0005576 & Extracellular region & 13 & 0.003785 \\
\hline GO:0030018 & $\mathrm{Z}$ disc & 4 & 0.006991 \\
\hline GO:0030141 & Secretory granule & 4 & 0.010644 \\
\hline \multicolumn{4}{|c|}{ molecular_function } \\
\hline GO:0048248 & CXCR3 chemokine receptor binding & 3 & $3.51 \mathrm{E}-08$ \\
\hline GO:0005525 & GTP binding & 15 & $2.48 \mathrm{E}-07$ \\
\hline GO:0003924 & GTPase activity & 13 & $3.24 \mathrm{E}-07$ \\
\hline GO:0008009 & Chemokine activity & 5 & $1.05 \mathrm{E}-06$ \\
\hline GO:0045236 & CXCR chemokine receptor binding & 3 & $3.30 \mathrm{E}-06$ \\
\hline GO:0042288 & MHC class I protein binding & 3 & $6.49 \mathrm{E}-05$ \\
\hline GO:0003779 & Actin binding & 9 & $9.09 \mathrm{E}-05$ \\
\hline GO:0005506 & Iron ion binding & 7 & 0.00032 \\
\hline GO:0004867 & Serine-type endopeptidase inhibitor activity & 5 & 0.000333 \\
\hline GO:0051879 & Hsp90 protein binding & 3 & 0.000451 \\
\hline
\end{tabular}

(MF) were related to retinoic acid-responsive element binding, ubiquitin binding, ATPase activity, and sequencespecific DNA activity (Figure 5(b)).

KEGG analysis revealed the potential mechanism of DEL and DEC in the SD-IR group (Figures 5(c) and 5(d)). Namely, DEL are involved in the regulation of dilated cardiomyopathy (DCM), hypertrophic cardiomyopathy (HCM), insulin signaling pathway, and mTOR signaling pathway (Figure 5(c)). The parent gene of DEC might take part in MAPK signaling pathway, cGMP-PKG signaling pathway, protein processing in endoplasmic reticulum, and adrenergic signaling in cardiomyocytes (Figure 5(d)).

3.7. Verification of Differentially Dysregulated $m R N A s$ and lncRNAs. We focused on the differentially dysregulated mRNAs and lncRNAs with more significant changes.
Compared with the IR group, the lncRNAs selected in the SD-IR group, including NONRATT012797.2 and NONRATT029190.2, were significantly overexpressed and consistent with the RNA-sequencing results (Figures 6(a) and $6(\mathrm{c})$ ). The lncRNAs selected in the SD-IR group, including NONRATT000247.2, NONRATT004098.2 and NONRATT025664.2, were significantly downregulated compared with the control group and were consistent with the RNAsequencing results (Figures 6(a) and 6(c)).

For further research, we selected three upregulated mRNAs (Ubd, Ccl12, Cxcl10) and two downregulated mRNAs (LOC100912599, Dpep1) (Figures 6(b) and 6(c)) in the SD-IR group for RT-qPCR verification, $p$ value $<0.05$, fold change $\geq 2$. The primers of mRNAs and lncRNAs are listed in Table 1 . Therefore, these results proved the accuracy of the microarray results. 
TABle 9: The Kyoto Encyclopedia of Genes and Genomes (KEGG) pathways enriched for the differentially expressed genes.

\begin{tabular}{|c|c|c|c|}
\hline Pathway ID & Term & Gene number & Pvalue \\
\hline rno04960 & Aldosterone-regulated sodium reabsorption & 4 & $2.24 \mathrm{E}-05$ \\
\hline rno04978 & Mineral absorption & 4 & 4.64E-05 \\
\hline rno05322 & Systemic lupus erythematosus & 5 & 0.000233 \\
\hline rno04614 & Renin-angiotensin system & 3 & 0.000307 \\
\hline rno04060 & Cytokine-cytokine receptor interaction & 8 & 0.00035 \\
\hline rno05145 & Toxoplasmosis & 5 & 0.000464 \\
\hline rno04621 & NOD-like receptor signaling pathway & 6 & 0.000495 \\
\hline rno04657 & IL-17 signaling pathway & 4 & 0.001424 \\
\hline rno05144 & Malaria & 3 & 0.00156 \\
\hline rno04972 & Pancreatic secretion & 4 & 0.00207 \\
\hline rno04668 & TNF signaling pathway & 4 & 0.002791 \\
\hline rno04750 & Inflammatory mediator regulation of TRP channels & 4 & 0.003408 \\
\hline rno04976 & Bile secretion & 3 & 0.003981 \\
\hline rno05133 & Pertussis & 3 & 0.004186 \\
\hline rno05202 & Transcriptional misregulation in cancers & 5 & 0.004801 \\
\hline rno04062 & Chemokine signaling pathway & 5 & 0.004938 \\
\hline rno04970 & Salivary secretion & 3 & 0.00532 \\
\hline rno00830 & Retinol metabolism & 3 & 0.006364 \\
\hline rno04650 & Natural killer cell mediated cytotoxicity & 4 & 0.00852 \\
\hline rno04974 & Protein digestion and absorption & 3 & 0.008842 \\
\hline
\end{tabular}

3.8. Construction of the $m R N A-m i R N A-\ln c R N A / \operatorname{circ} R N A$ Network. CircRNA participates in the regulation of biological processes in different ways. It is well known that circRNA contains multiple binding sites of miRNA and is also regulated by miRNA. Analysis of circRNA-miRNA interaction may clarify the function and mechanism of circRNA.

As shown in Figure 7(a), the network involves 30 lncRNAs, 35 mRNAs, and 13 miRNAs. At the same time, a circRNA-miRNA-mRNA ceRNA network was constructed (Figure 7(b)), involving 26 circRNAs, 44 mRNAs, and 16 miRNAs. Each differentially expressed lncRNA can be associated with one or more miRNAs. For example, lncRNA NONRATT016892.2 has established connections with two miRNAs, including rno-miR-1-3p and rno-miR-206-3p. miR1187 is connected with four circRNAs, including circRNA_ 02339/Chr12:587083_591296, circRNA_07789/Chr4:58661995_58669806, circRNA_04050/Chr16:23555919_23573530, and circRNA_07510/Chr3:175512077_175535023. Finally, circRNA_01445/Chr10:37180938_37185721 has only established a connection with miR-438 (Figure 7(b)).

The two networks have multiple common nodes (Figure 7(c)), such as lncRNA NONRATT024121.2, lncRNA NONRATT022775.2, lncRNA NONRATT022692.2, lncRNA NONRATT011191.2, and lncRNA NONRATT017402.2, which all interact with miR-493-5p.

3.9. PPI Network and Functional Analysis of the Differentially Expressed $m R N A s$. To further address the most significant clusters of differentially expressed mRNAs in the ceRNA network, we conducted the PPI network analysis by using the STRING database version 11.0 and visualization under the Cytohubba plug-in and the Cytoscape. The most significant hub upregulated genes in the PPI network were Cxcl10, Cxcl11, Mmp9, Gbp2, Gbp5, Irgm, Mpa21, and Igf1, while the most significant hub downregulated genes were Ahsg, Trim63, and Trpv4 (Figure 8(a)).

To clarify the role of differential genes in the preventive effect of cardiac sympathetic denervation on MIRI, we performed GO and KEGG analyses on the differentially expressed mRNAs. The results suggested that the molecular functions (MF) are mainly enriched in the CXCR3 chemokine receptor binding, MHC class I protein binding, GTP binding, GTPase activity, and chemokine activity (Figure 8(b)). In the cell components (CC), functions are highly enriched in autophagy-related processes, which are related to the cortical cytoskeleton, nucleosomes, secretory granules. KEGG analysis showed that the differentially expressed mRNAs were involved in cytokine-cytokine receptor interaction, NOD-like receptor signaling pathway, chemokine signaling pathway, and inflammatory mediator regulation of TRP channels (Figure $8(\mathrm{c})$ ). These results showed that most of the hub genes play a role in the preventive effect of cardiac sympathetic denervation on MIRI.

\section{Discussion}

This study provides novel information on the vital role of cardiac sympathetic denervation in the process of myocardial ischemia/reperfusion injury. Our main findings are as follows: (1) Cardiac sympathetic denervation induced by 6OHDA alleviated myocardial ischemia/reperfusion injury. (2) The expression profiles of lncRNA, circRNA, and mRNA in the upper thoracic spinal cord were identified by RNA-seq analysis. Among them, there were 148 upregulated and 51 
LncRNA

SD_IR-vs-IR(total): top 30 GO term

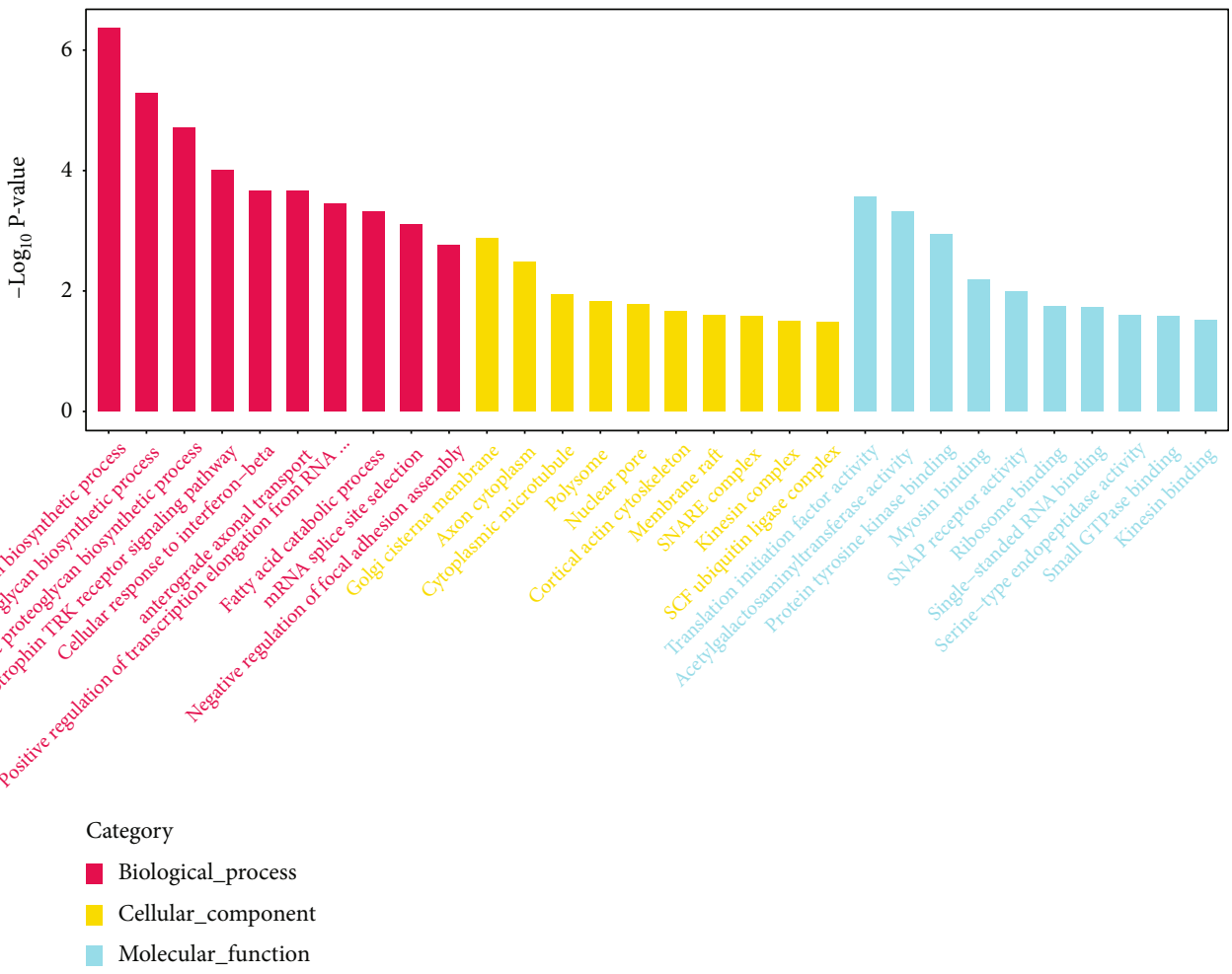

(a)

CircRNA

SD_IR-vs-IR (total): top $30 \mathrm{GO}$ term

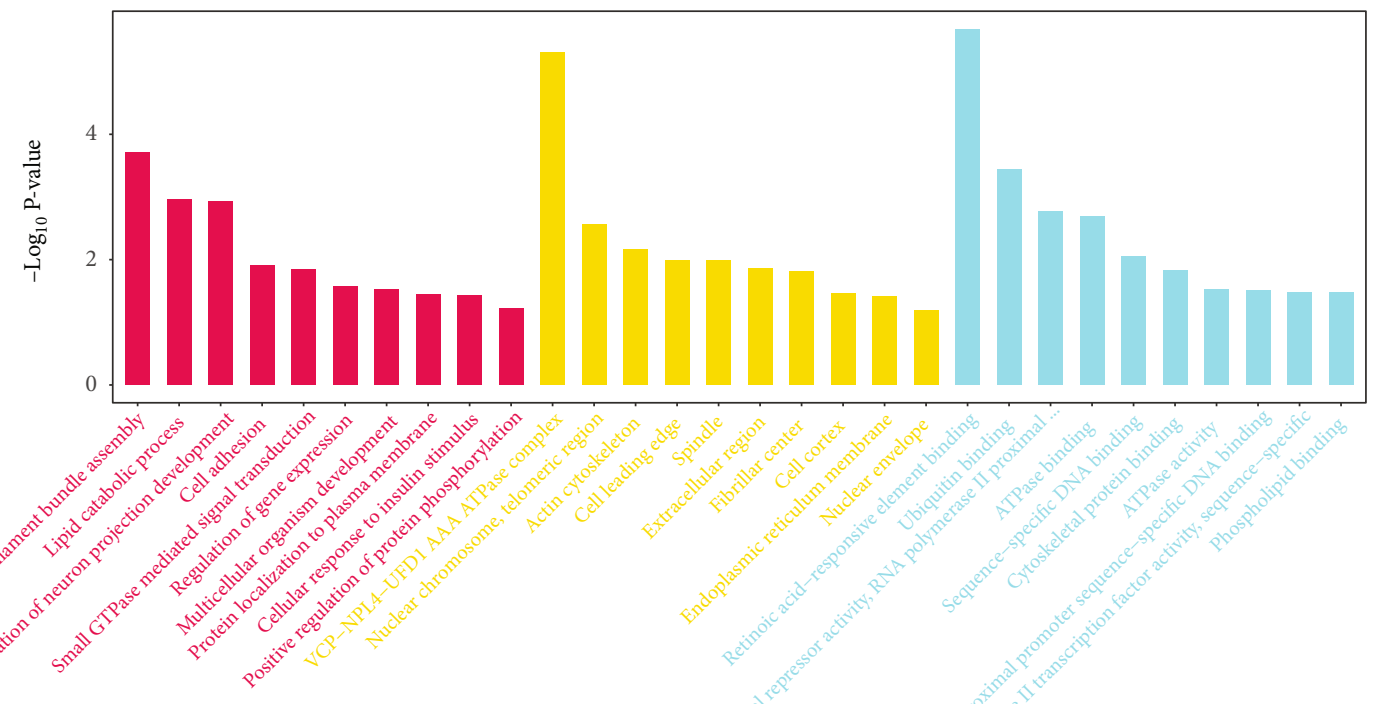

Category

- Biological_process

Cellular_component

Molecular_function

(b)

Figure 5: Continued. 


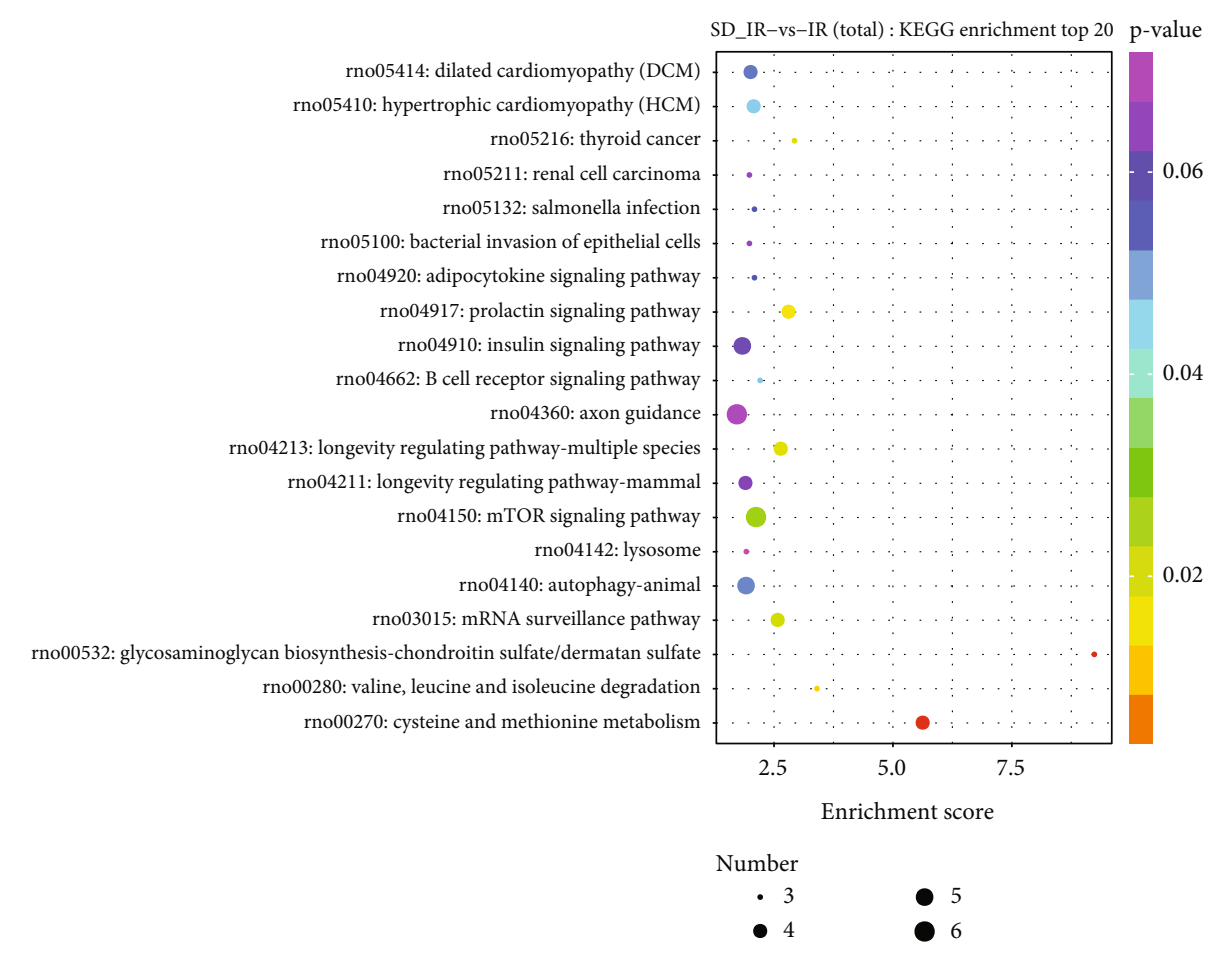

(c)

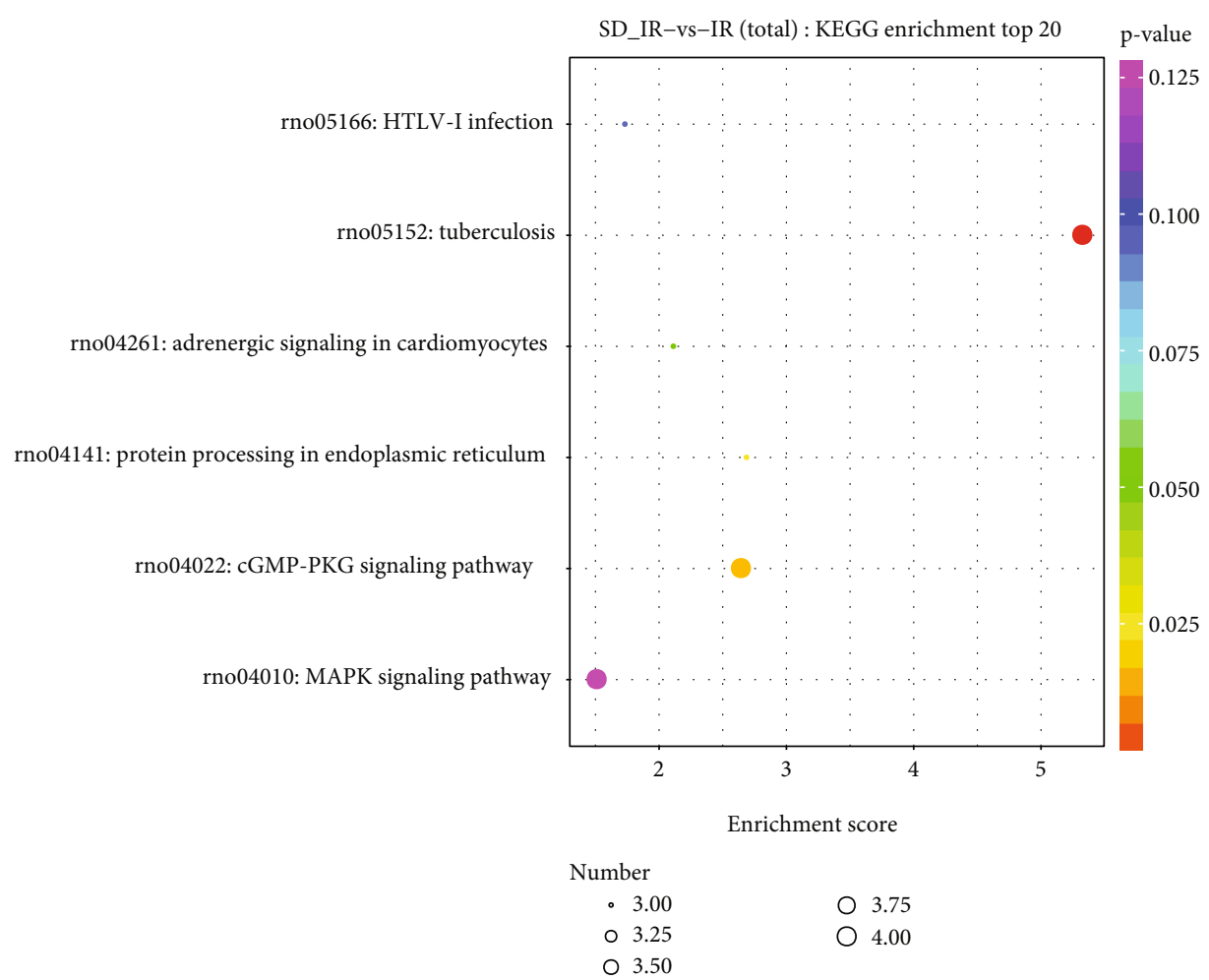

(d)

FIgURE 5: Functional analysis of the differentially expressed lncRNAs and circRNAs between IR group and SD-IR group. (a) The top 30 Gene Ontology terms of differentially expressed lncRNAs. Green color is related to biological processes; blue color is related to cellular components; and red color is related to molecular functions. (b) The top 30 Gene Ontology terms of differentially expressed circRNAs. Richly factor refers to the ratio of the number of differentially expressed genes in the KEGG pathway accounting for the total number of genes that are related to this pathway. The larger the richly factor, the higher the degree of enrichment; the size of the bubble indicates the number of genes, which is qualified by Q-value. (c) The top 20 KEGG pathway enrichment analysis of differentially expressed lncRNAs. (d) The top six KEGG pathway enrichment analysis of differentially expressed circRNAs. 

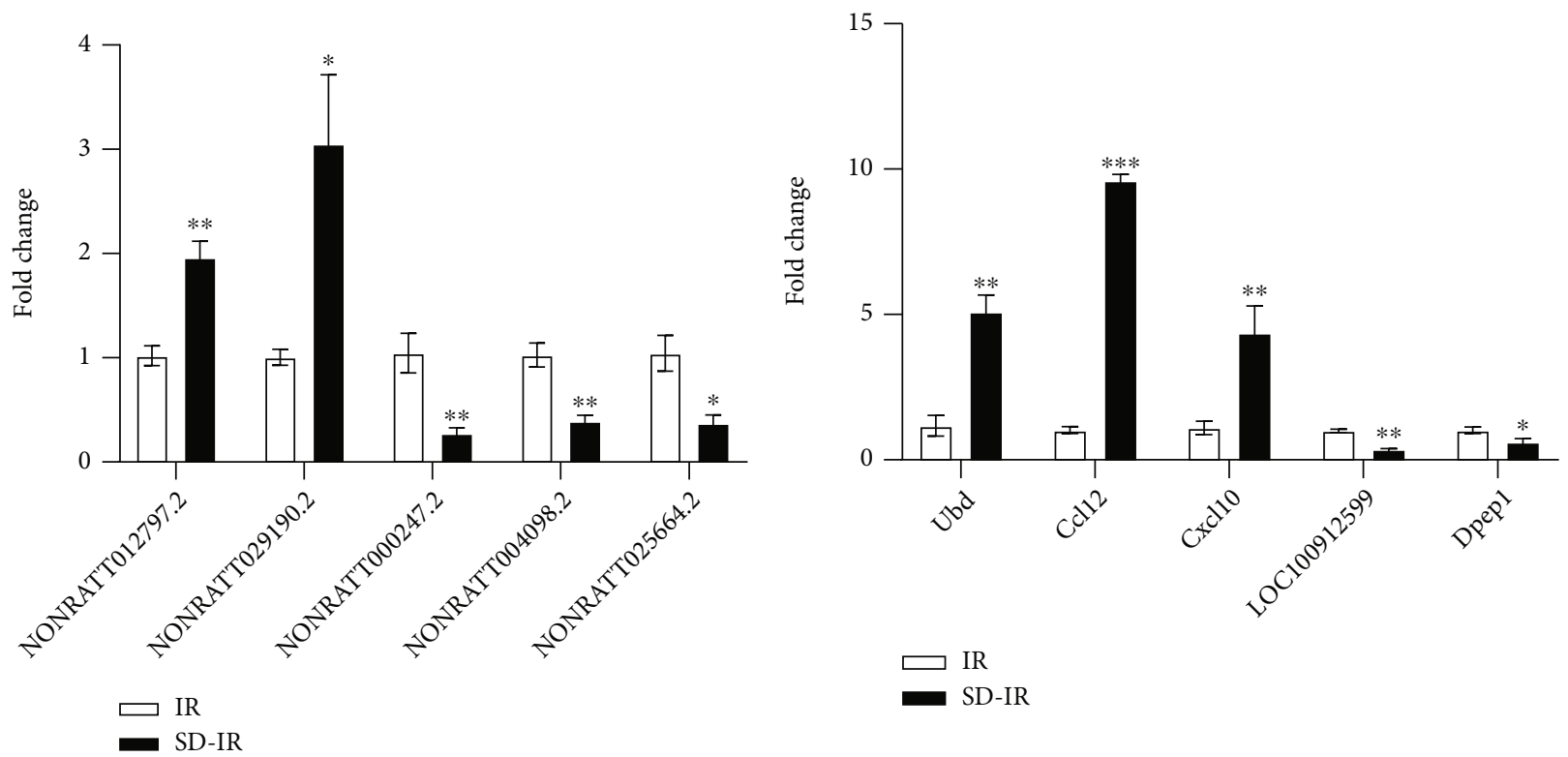

(a)

(b)

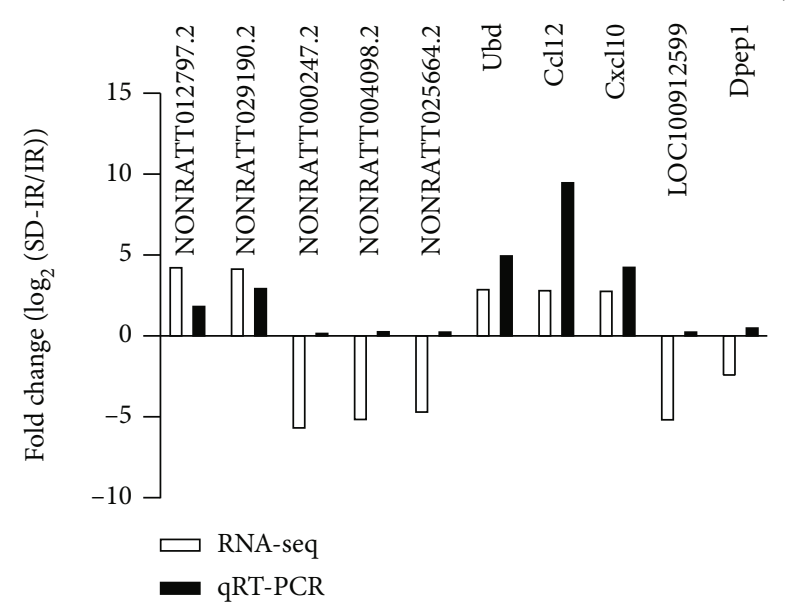

(c)

FIGURE 6: Validation of lncRNAs and mRNAs by RT-qPCR in the T1-T4 spinal cord IR group and SD-IR group. (a) The expression levels of lncRNA NONRATT012797.2 and NONRATT029190.2 were significantly upregulated in SD-IR group, whereas the expression levels of lncRNA NONRATT000247.2, NONRATT004098.2, and NONRATT025664.2 were significantly downregulated in SD-IR group. (b) The expression levels of mRNA Ubd, Ccl12, and Cxcl10 were significantly upregulated in SD-IR group, whereas the expression levels of mRNA LOC100912599 and Dpep1 were significantly downregulated in SD-IR group. (c) The expression levels of five lncRNAs and five mRNAs. Two upregulated lncRNAs, three downregulated lncRNAs, three upregulated mRNAs, and two downregulated mRNAs were validated by RT-qPCR. Data are expressed as mean \pm SEM. $* P<0.05, * * \mathrm{p}<0.01, * * * \mathrm{p}<0.001$ vs. IR group.

downregulated mRNAs, 165 upregulated and 168 downregulated lncRNAs, and 70 upregulated and 52 downregulated circRNAs in the SD-IR group compared with the IR group. (3) We selected three mRNAs from the most upregulated mRNAs and three lncRNAs from the most downregulated lncRNAs for RT-qPCR low-throughput verification, and the results were consistent with the sequencing results. By providing new insights into the function of lncRNA/circRNA-miRNAmRNA networks, our results contribute to the understanding of the pathogenesis of MIRI and provide new targets for MIRI.

In recent years, a large number of studies have confirmed that cardiac sympathetic activity plays an important role in many cardiac diseases and processes [35-40]. Lu et al. reported that sympathetic hyperinnervation and/or myocardial infarction remodeled myocardial glutamate signaling and ultimately increased the severity of ventricular tachyarrhythmias [9]. It has also been shown that left stellate ganglion (LSG) suppression protects against ventricular arrhythmias. Yu et al. found that optogenetic modulation could reversibly inhibit the neural activity of LSG, thereby increasing electrophysiological stability and protecting against myocardial ischemia-induced ventricular arrhythmias [41]. These reports and our results also suggest that the presence of decreased cardiac sympathetic activity can have a cardioprotective effect, and that this depends on effective sympathetic denervation. 

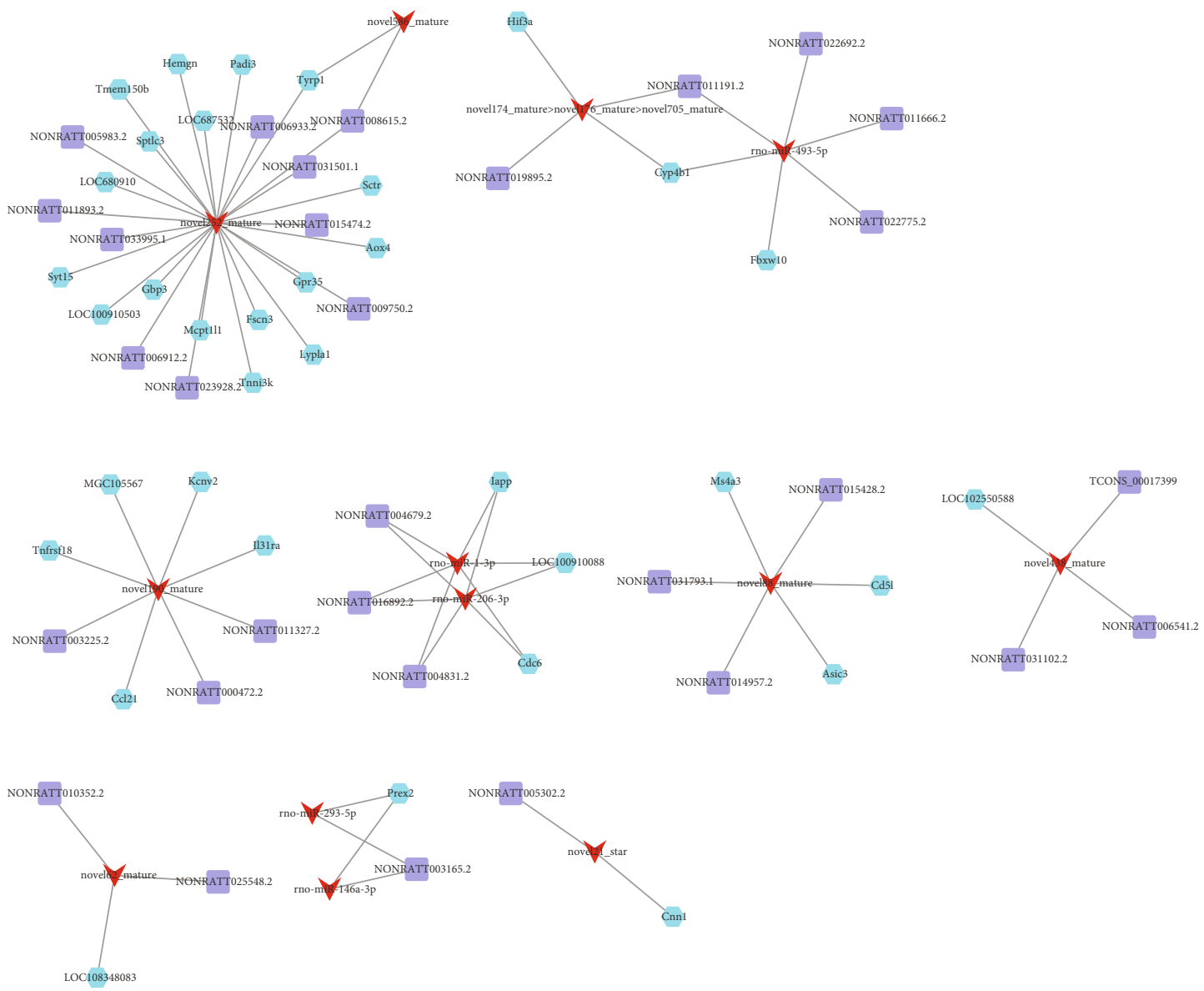

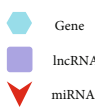

(a)

FIgUre 7: Continued. 

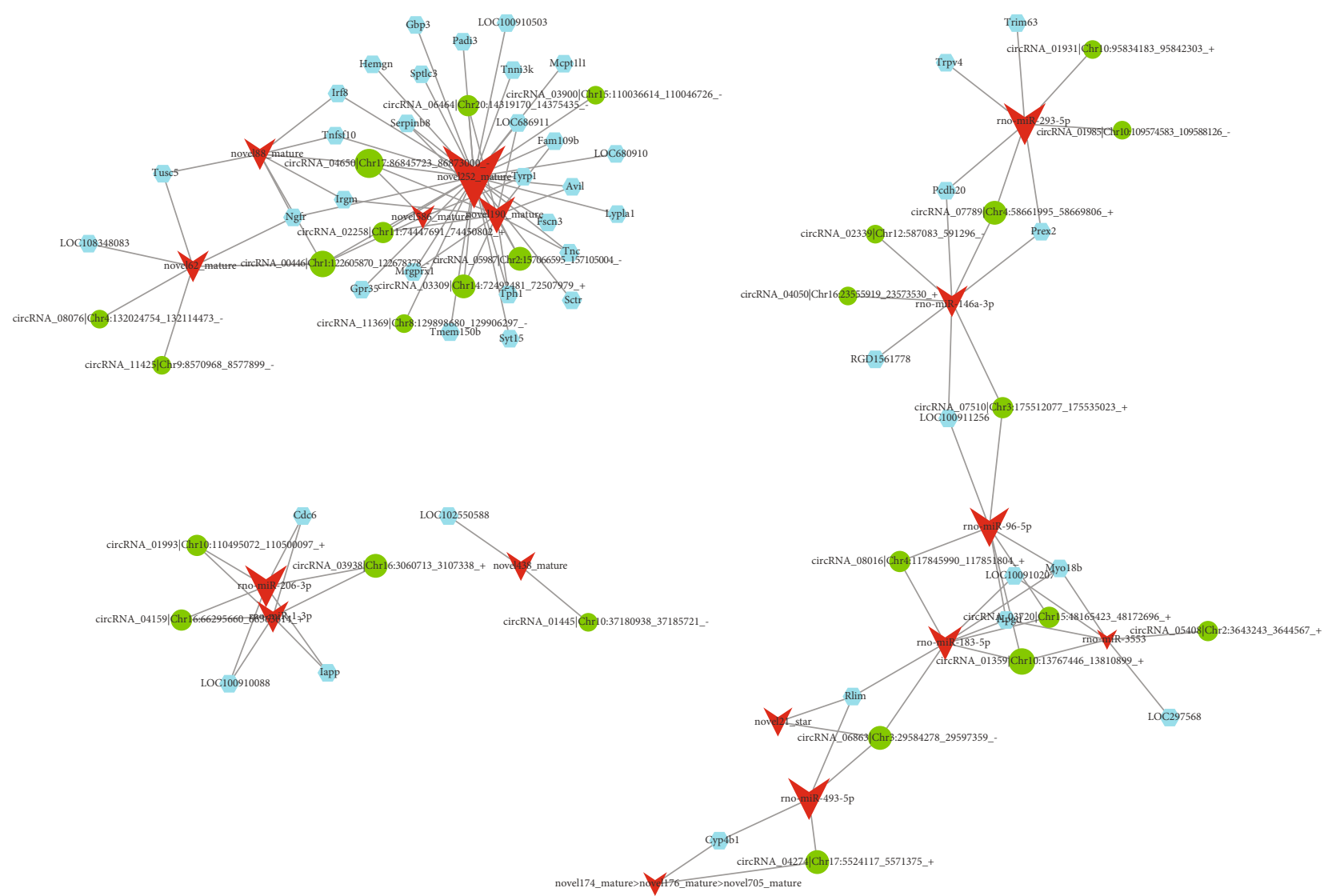

circRNA

$\checkmark$ miRNA

gene

(b)

Figure 7: Continued. 


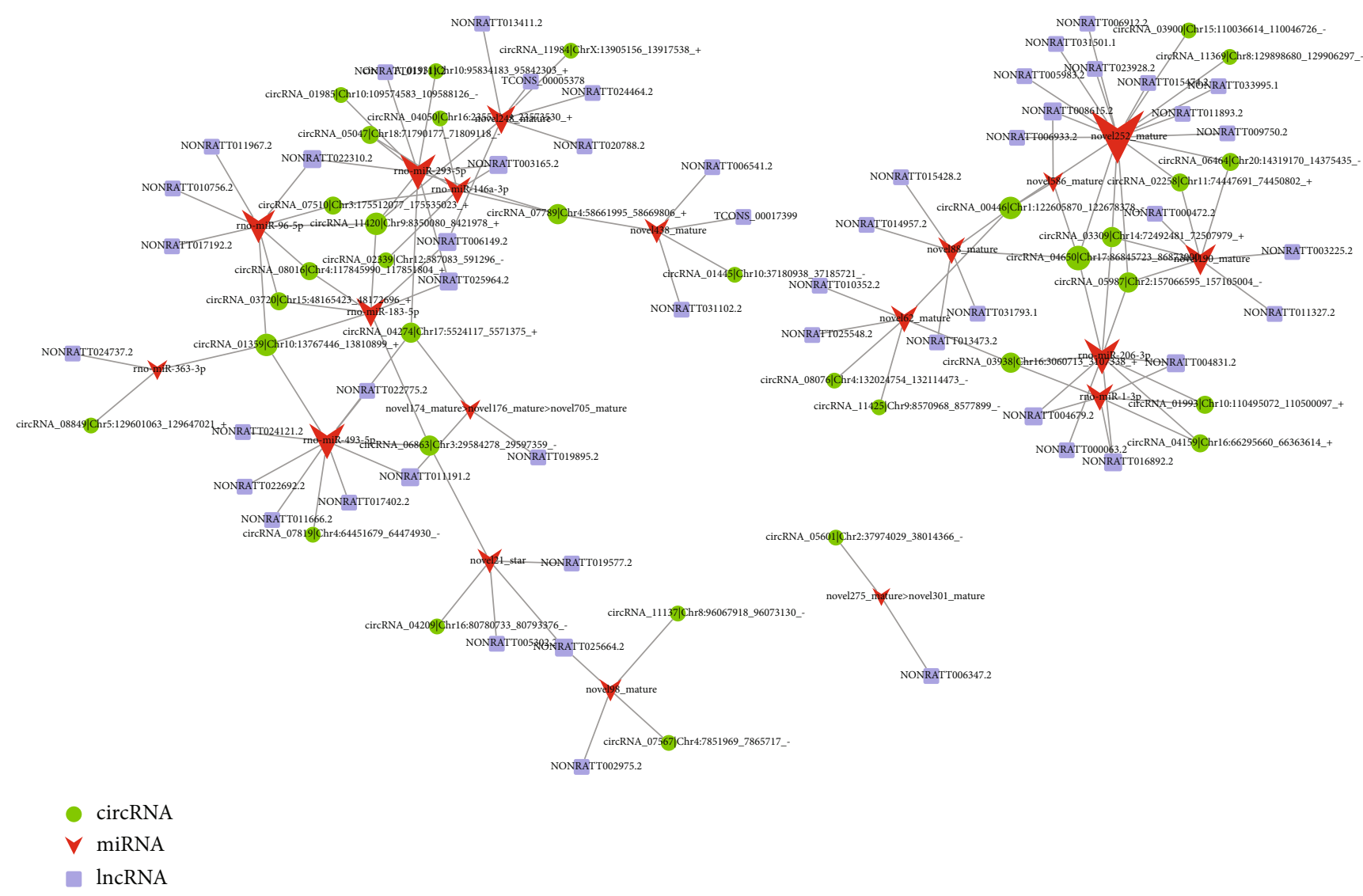

(c)

FIgURE 7: Construction of the mRNA-miRNA-lncRNA/circRNA network. (a) Network analysis of mRNA-miRNA-lncRNA. The blue nodes represent mRNA. The purple nodes represent lncRNA. The red nodes represent miRNA. (b) Network analysis of mRNA-miRNA-circRNA. The green nodes represent circRNA. The red nodes represent miRNA. The blue nodes represent mRNA. (c) Network analysis of lncRNAmiRNA-circRNA. The green nodes represent circRNA. The red nodes represent miRNA. The purple nodes represent lncRNA.

Recently, significant efforts have been made to understand the alterations of ncRNAs in different spinal cord segments and their contributions to specific outcomes of diseases $[16,26,42-44]$. The spinal cord is a complex and dynamic neural structure. It contains sympathetic preganglionic neurons within the intermediolateral cell column [45-47]; they are involved in the generation of sympathetic activity in many autonomic targets, including the heart and blood vessels [36, 48-50]. There is accumulating evidence of the interaction between the spinal cord and the heart [51-55]. We previously demonstrated the changes of novel lncRNAs in the upper thoracic spinal cord of rats with MIRI [42]. In recent years, there has been considerable effort to explore the relationship between cardiac sympathetic activity and cardiovascular diseases. However, the changes in spinal lncRNAs in rats with MIRI after cardiac sympathetic denervation have not been reported. Here, we aimed to understand the involvement of specific patterns of changes in the lncRNA/circRNAs-miRNA-mRNA network of the upper thoracic spinal cord regions of animals with myocardial ischemia-reperfusion injury after cardiac sympathetic denervation.

LncRNAs are involved in the progression of coronary artery disease (CAD) [56]. Xu et al. reported that lncRNA
AC096664.3/PPAR-gamma/ABCG1-dependent signal transduction pathway contributes to the regulation of cholesterol homeostasis [56]. As one of the differentially expressed lncRNAs between CAD patients and healthy controls, lncRNA ENST00000602558.1 plays a key role in the pathogenesis of atherosclerosis. Cai et al. showed that lncRNA ENST00000602558.1 regulated ABCG1 expression and cholesterol efflux from vascular smooth muscle cells through a p65-dependent pathway [57]. The study by Li et al. provided the characterization of lncRNA expression profile and identification of novel lncRNA biomarkers to diagnose CAD [58]. According to our study, spinal lncRNA as a sponge of miRNA mainly participates in the process of MIRI through cysteine and methionine metabolism, mTOR signaling pathway, insulin signaling pathway, and adipocytokine signaling pathway.

Circular RNAs (circRNAs) play a critical role in the physiology and pathology of cardiovascular diseases [59-62]. To further investigate the roles of these differentially expressed circRNAs in the development of MIRI, we performed GO and KEGG pathway analyses. Based on the GO and KEGG enrichment analyses of these circRNAs, our results suggested that the significantly enriched biologic processes and molecular functions of the upregulated genes after MIRI were associated with gene sets termed as follows: 


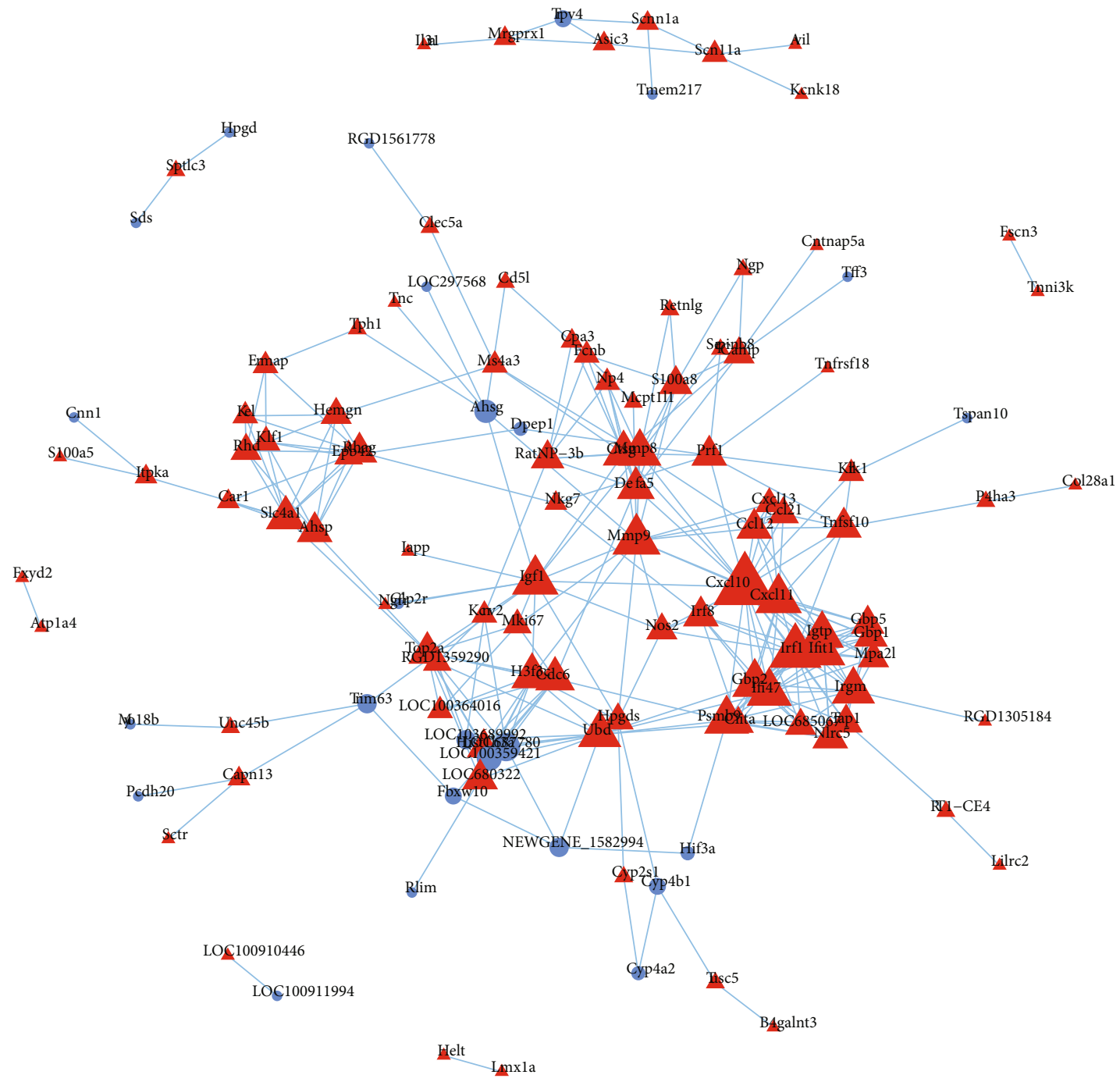

(a)

Figure 8: Continued. 
SD_IR-vs-IR (total) : top $30 \mathrm{GO}$ term

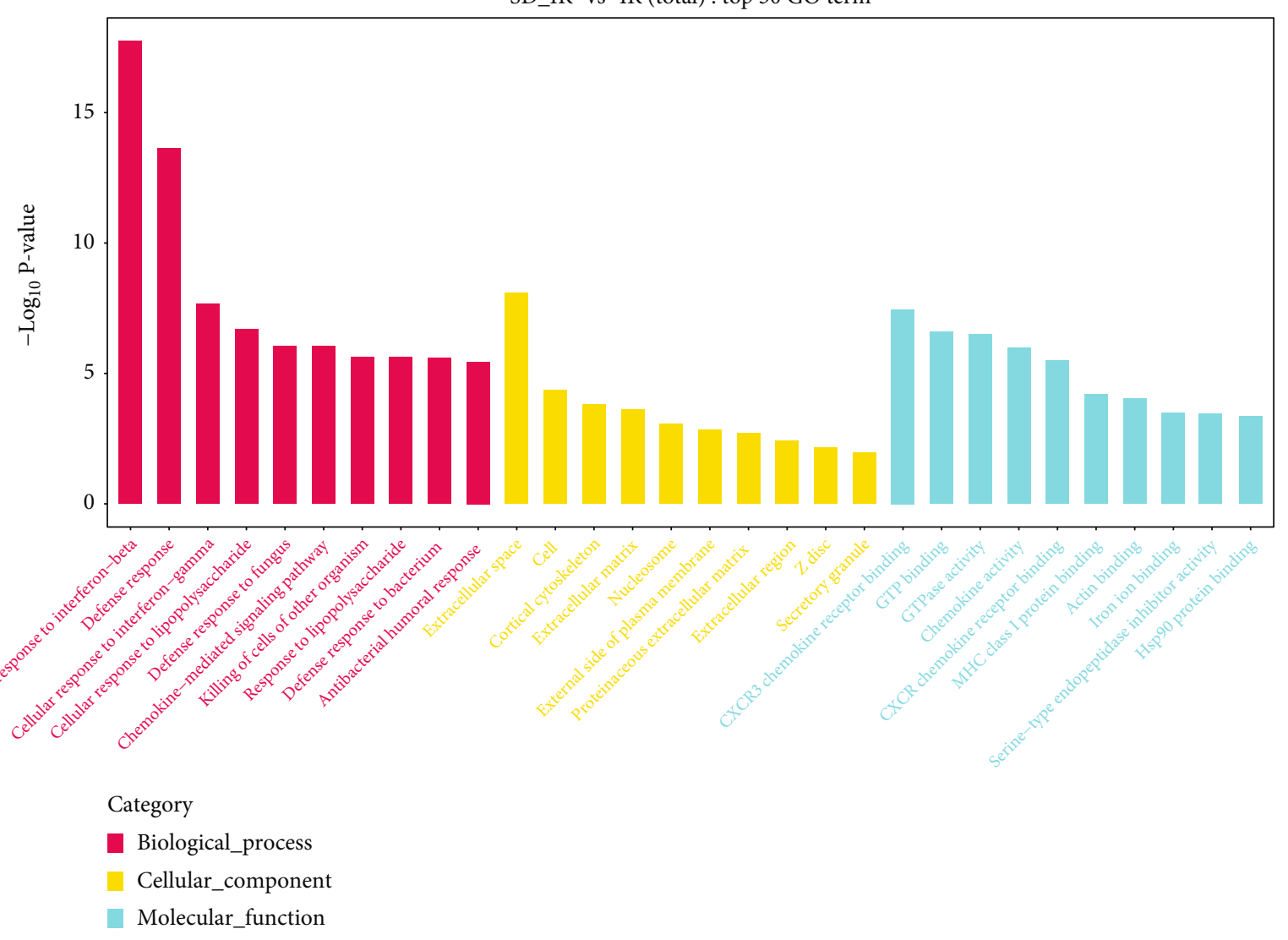

(b)

Figure 8: Continued. 


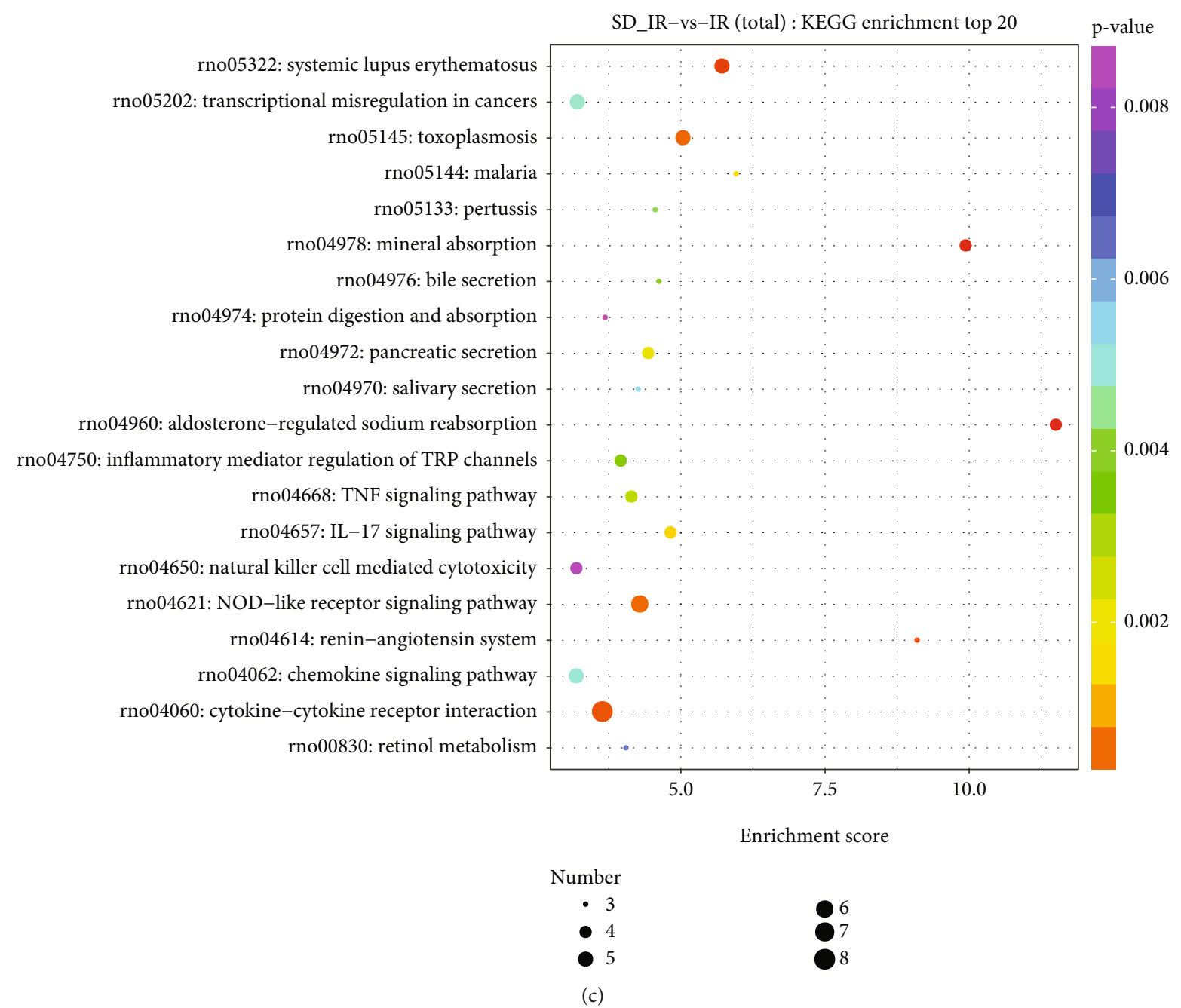

FIGURE 8: PPI network and functional analysis of the differentially expressed mRNAs. (a) The PPI network was constructed by differentially expressed mRNAs. (b) The top 30 Gene Ontology terms of differentially expressed mRNAs. (c) The top 20 KEGG pathway enrichment analysis of differentially expressed mRNAs.

"MAPK signaling pathway" and "cGMP-PKG signaling pathway". It is well known that MAPK pathway is involved in ischemia-reperfusion injury [63]. Previous studies have shown that CGMP-PKG pathways are implicated in cardiovascular complications of diverse etiologies [64, 65]. These data suggest that spinal circRNAs may be potential targets for MIRI.

miRNAs have been shown to modulate the translational activity of the genome and regulate protein expression and function [66-68]. According to Wang et al. [69], miRNA493-5p promotes apoptosis and suppresses proliferation and invasion in liver cancer cells by targeting VAMP2. Previous studies have pointed out a potential cardioprotective role of phosphatidylserine in heart ischemia [70-73], suggesting that the phosphatidylserine signaling pathway is associated with MIRI. Schumacher et al. [74] indicated that phosphatidylserine significantly reduced the infarct size by $30 \%$ and improved heart function by $25 \%$ in a chronic model of acute myocardial infarction (AMI), suggesting that phosphatidylserine supplementation may be a promising novel strategy to reduce infarct size following AMI and to prevent myocardial injury during myocardial infarction or cardiac surgery. A large number of studies have confirmed that chemokines [75-77], including C-X-C motif chemokine receptor 3 (CXCR3) [78], are closely related to the ischemiareperfusion injury. In this study, we found that miRNAs in the spinal cord participated in the molecular progression of MIRI through the regulation of actin cytoskeleton, phospholipase D, calcium, and MAPK signaling pathways.

It has been found that the lncRNA/circRNA-miRNAmRNA ceRNA network plays a role in multiple physiological and pathological processes [66, 79-86]. Cheng et al. [82] reported the comprehensive analysis of the circRNAlncRNA-miRNA-mRNA ceRNA network in the prognosis of acute myeloid leukemia (AML), elucidated the posttranscriptional regulatory mechanism of AML, and identified novel AML prognostic biomarkers, which has important guiding significance for the clinical diagnosis, treatment, and further scientific research of AML. Wang et al. [87] established bronchopulmonary dysplasia (BPD)-related ceRNA 
regulatory network of circRNA/lncRNA-miRNA-mRNA in the lung tissue of a mouse model, proving that it is significantly associated with the pathophysiological characteristics of BPD. In this study, we analyzed the changes in spinal lncRNA-miRNA-mRNA and circRNA-miRNAmRNA ceRNA networks in MIRI after cardiac sympathetic denervation. Our findings offer a new direction for understanding the pathogenesis of MIRI, and suggest some effective targets in the spinal cord after cardiac sympathetic denervation.

In conclusion, the expression characteristics of coding genes, miRNAs, lncRNAs, and circRNAs in the upper thoracic spinal cord of MIRI rats were determined after cardiac sympathetic denervation induced by 6-OHDA. The preventive effect of cardiac sympathetic denervation on MIRI paves the road for further studies on the sympathetic mechanisms associated with MIRI, which is important to further explore the pathogenesis of MIRI and potentially facilitate the discovery of novel lncRNA/circRNA-miRNA-mRNA networks for therapeutic targeting in the management of MIRI.

\section{Data Availability}

The raw sequencing data presented in this paper have been deposited in the Sequence Read Archive (SRA) under accession number PRJNA776390. The records are accessible with the following link: https://www.ncbi.nlm.nih.gov/sra/ PRJNA776390.

\section{Ethical Approval}

The animal study was reviewed and approved by the Institutional Animal Care and Use Committee of Tongji Hospital, Tongji Medical College, Huazhong University of Science and Technology, Wuhan, China (IRB ID: TJ-A0804).

\section{Conflicts of Interest}

The authors declare that this study received assistance from OEbiotech Co. Ltd. The OEbiotech Co. Ltd. was not involved in the study design, collection, analysis, interpretation of data, the writing of this article, or the decision to submit it for publication.

\section{Authors' Contributions}

All authors listed have made a substantial, direct, and intellectual contribution to the work and approved it for publication.

\section{Funding}

This project was supported by grants from the National Natural Science Foundation of China (No. 81670240 and No. 81873467), the National Natural Science Foundation of Hubei Province (No. 2016CFB625), the Medical Innovation Project in Fujian Province (No. 2017-CX-48), and the Key Research and Development Project of Hainan Province (ZDYF2021SHFZ087).

\section{Acknowledgments}

We thank the OEbiotech Co. Ltd. (Shanghai, China) for providing assistance with the data analysis of $16 \mathrm{~S}$ rRNA sequencing. We thank LetPub (http://www.letpub.com) for its linguistic assistance during the preparation of this manuscript.

\section{References}

[1] J. Bai, C. Ren, W. Hao, R. Wang, and J. M. Cao, "Chemical sympathetic denervation, suppression of myocardial transient outward potassium current, and ventricular fibrillation in the rat," Canadian Journal of Physiology and Pharmacology, vol. 86, no. 10, pp. 700-709, 2008.

[2] Q. Wang, Z. G. He, S. Y. Li, M. H. Feng, and H. B. Xiang, "Application of animal and human PET in cardiac research," American journal of cardiovascular disease, vol. 8, no. 3, pp. 24-30, 2018.

[3] X. C. Pan, Z. X. Li, D. Z. Wu, S. Y. Li, H. B. Xiang, and Y. T. Song, "Mapping changes of whole brain blood flow in rats with myocardial ischemia/reperfusion injury assessed by positron emission tomography," Current Medical Science, vol. 39, no. 4, pp. 653-657, 2019.

[4] M. Chen, Z. G. He, B. W. Liu, Z. X. Li, S. G. Liu, and H. B. Xiang, "Parafascicular nucleus-heart neural crosstalk: implications for seizure- induced myocardial stunning," Epilepsy \& Behavior, vol. 63, pp. 135-137, 2016.

[5] C. Y. Li and Y. G. Li, "Cardiac sympathetic nerve sprouting and susceptibility to ventricular arrhythmias after myocardial infarction," Cardiology Research and Practice, vol. 2015, 8 pages, 2015.

[6] B. A. Habecker, M. E. Anderson, S. J. Birren et al., "Molecular and cellular neurocardiology: development, and cellular and molecular adaptations to heart disease," The Journal of Physiology, vol. 594, no. 14, pp. 3853-3875, 2016.

[7] J. H. Coote and R. A. Chauhan, "The sympathetic innervation of the heart: important new insights," Autonomic Neuroscience, vol. 199, pp. 17-23, 2016.

[8] R. F. Gilmour, "Life out of balance: the sympathetic nervous system and cardiac arrhythmias," Cardiovascular Research, vol. 51, no. 4, pp. 625-626, 2001.

[9] J. Lu, X. Gao, J. Gu et al., "Nerve sprouting contributes to increased severity of ventricular tachyarrhythmias by upregulating iGluRs in rats with healed myocardial necrotic injury," Journal of Molecular Neuroscience, vol. 48, no. 2, pp. 448455, 2012.

[10] N. Herring, J. Cranley, M. N. Lokale et al., "The cardiac sympathetic co-transmitter galanin reduces acetylcholine release and vagal bradycardia: implications for neural control of cardiac excitability," Journal of Molecular and Cellular Cardiology, vol. 52, no. 3, pp. 667-676, 2012.

[11] M. Palkeeva, I. Studneva, A. Molokoedov et al., "Galanin/GalR1-3 system: a promising therapeutic target for myocardial ischemia/reperfusion injury," Biomedicine \& Pharmacotherapy, vol. 109, pp. 1556-1562, 2019.

[12] M. T. Foster and T. J. Bartness, "Sympathetic but not sensory denervation stimulates white adipocyte proliferation," American Journal of Physiology-Regulatory, Integrative and Comparative Physiology, vol. 291, no. 6, pp. R1630-R1637, 2006. 
[13] M. A. Samuels, "The brain-heart connection," Circulation, vol. 116, no. 1, pp. 77-84, 2007.

[14] A. T. Mazzeo, A. Micalizzi, L. Mascia, A. Scicolone, and L. Siracusano, "Brain-heart crosstalk: the many faces of stress-related cardiomyopathy syndromes in anaesthesia and intensive care," British Journal of Anaesthesia, vol. 112, no. 5, pp. 803-815, 2014.

[15] Q. Wang, Z. X. Li, Y. J. Li et al., "Alterations in amino acid levels and metabolite ratio of spinal cord in rat with myocardial ischemia-reperfusion injury by proton magnetic resonance spectroscopy," American Journal of Translational Research, vol. 11, p. 3101, 2019.

[16] Q. Wang, Z. X. Li, Y. J. Li et al., "Identification of lncRNA and mRNA expression profiles in rat spinal cords at various time-points following cardiac ischemia/reperfusion," International Journal of Molecular Medicine, vol. 43, no. 6, pp. 2361-2375, 2019.

[17] S. Y. Li, Z. X. Li, Z. G. He et al., "Quantitative proteomics reveal the alterations in the spinal cord after myocardial ischemia-reperfusion injury in rats," International Journal of Molecular Medicine, vol. 44, no. 5, pp. 1877-1887, 2019.

[18] S. Hombach and M. Kretz, "Non-coding RNAs: classification, biology and functioning," Advances in Experimental Medicine and Biology, vol. 937, pp. 3-17, 2016.

[19] J. S. Mattick and I. V. Makunin, "Non-coding RNA," Human Molecular Genetics, vol. 15, no. 1, pp. R17-R29, 2006.

[20] C.-H. Jung, M. A. Hansen, I. V. Makunin, D. J. Korbie, and J. S. Mattick, "Identification of novel non-coding RNAs using profiles of short sequence reads from next generation sequencing data," Genomics, vol. 11, no. 1, 2010.

[21] B. Wang, J. Wu, Q. L. Huang et al., "Comprehensive analysis of differentially expressed lncRNA, circRNA and mRNA and their ceRNA networks in mice with severe acute pancreatitis," Frontiers in Genetics, vol. 12, p. 14, 2021.

[22] F. Kopp and J. T. Mendell, "Functional classification and experimental dissection of long noncoding RNAs," Cell, vol. 172, no. 3, pp. 393-407, 2018.

[23] M. Esteller, "Non-coding RNAs in human disease," Nature Reviews Genetics, vol. 12, no. 12, pp. 861-874, 2011.

[24] M. J. Picklo, "Methods of sympathetic degeneration and alteration," Journal of the Autonomic Nervous System, vol. 62, no. 3, pp. 111-125, 1997.

[25] P. Jiang, D. Ma, Y. Jiang et al., "Preventive effect of different compatibilities of Ramulus Cinnamomi and radix Paeomlae alba in Guizhi decoction on cardiac sympathetic denervation induced by 6-OHDA," Chinese Journal of Integrated Traditional and Western Medicine, vol. 36, no. 5, pp. 608-613, 2016.

[26] Q. Wang, Z. G. He, Z. X. Li et al., "Bioinformatics analysis of gene expression profile data to screen key genes involved in cardiac ischemia-reperfusion injury," International Journal of Clinical and Experimental Medicine, vol. 11, pp. 4955-4966, 2018.

[27] M. H. Feng, Z. X. Li, Q. Wang et al., "Neurochemical alterations of different cerebral regions in rats with myocardial ischemia-reperfusion injury based on proton nuclear magnetic spectroscopy analysis," Aging, vol. 13, no. 2, pp. 2294-2309, 2021.

[28] B. A. Boughton, D. L. Callahan, C. Silva et al., "Comprehensive profiling and quantitation of amine group containing metabolites," Analytical Chemistry, vol. 83, no. 19, pp. 7523-7530, 2011.
[29] J. Wang, L. Zhou, H. Lei et al., "Simultaneous quantification of amino metabolites in multiple metabolic pathways using ultrahigh performance liquid chromatography with tandem-mass spectrometry," Scientific Reports, vol. 7, no. 1, 2017.

[30] L. Gu, A. D. Jones, and R. L. Last, "LC-MS/MS assay for protein amino acids and metabolically related compounds for large-scale screening of metabolic phenotypes," Analytical Chemistry, vol. 79, no. 21, pp. 8067-8075, 2007.

[31] K. Dettmer, P. A. Aronov, and B. D. Hammock, "Mass spectrometry-based metabolomics," Mass Spectrometry Reviews, vol. 26, no. 1, pp. 51-78, 2007.

[32] Y. F. Cheng, Y. T. Chang, W. H. Chen et al., "Cardioprotection induced in a mouse model of neuropathic pain via anterior nucleus of paraventricular thalamus," Nature Communications, vol. 8, no. 1, p. 826, 2017.

[33] S. Bohl, D. J. Medway, J. Schulz-Menger, J. E. Schneider, S. Neubauer, and C. A. Lygate, "Refined approach for quantification of in vivo ischemia-reperfusion injury in the mouse heart," American Journal of Physiology-Heart and Circulatory Physiology, vol. 297, no. 6, pp. H2054-H2058, 2009.

[34] X. F. Liang, Y. C. Lai, W. Z. Wu et al., "LncRNA-miRNAmRNA expression variation profile in the urine of calcium oxalate stone patients," BMC Medical Genomics, vol. 12, no. 1, p. 57, 2019.

[35] J. A. Moffitt, "Role for neural growth factor in autonomically driven arrhythmogenesis? Focus on: "structural neuroplasticity following T5 spinal cord transection: increased cardiac sympathetic innervation density and SPN arborization"," American Journal of Physiology-Regulatory, Integrative and Comparative Physiology, vol. 299, no. 4, pp. R983-R984, 2010.

[36] H. L. Lujan, G. Palani, and S. E. DiCarlo, "Structural neuroplasticity following T5 spinal cord transection: increased cardiac sympathetic innervation density and SPN arborization," American Journal of Physiology-Regulatory, Integrative and Comparative Physiology, vol. 299, no. 4, pp. R985R995, 2010.

[37] L. Fan, B. Xiang, J. Xiong, Z. He, and H. B. Xiang, "Use of viruses for interrogating viscera-specific projections in central nervous system," Journal of Neuroscience Methods, vol. 341, p. 108757, 2020.

[38] M. Chen, L. Yu, X. Sheng, Q. Liu, H. Jiang, and S. Zhou, "Cardiac autonomic tone modulators: promising feasible options for heart failure with hyper-sympathetic activity," International Journal of Cardiology, vol. 198, pp. 185-186, 2015.

[39] Y. Lai, L. Yu, and H. Jiang, "Autonomic neuromodulation for preventing and treating ventricular arrhythmias," Frontiers in Physiology, vol. 10, 2019.

[40] G. Meng, X. Zhou, M. Wang et al., "Gut microbe-derived metabolite trimethylamine $\mathrm{N}$-oxide activates the cardiac autonomic nervous system and facilitates ischemia-induced ventricular arrhythmia via two different pathways," eBioMedicine, vol. 44, pp. 656-664, 2019.

[41] L. Yu, L. Zhou, G. Cao et al., "Optogenetic modulation of cardiac sympathetic nerve activity to prevent ventricular arrhythmias," Journal of the American College of Cardiology, vol. 70, no. 22, pp. 2778-2790, 2017.

[42] Z. X. Li, Y. J. Li, Q. Wang, Z. G. He, M. H. Feng, and H. B. Xiang, "Characterization of novel lncRNAs in upper thoracic spinal cords of rats with myocardial ischemia-reperfusion injury," Experimental and Therapeutic Medicine, vol. 21, p. $15,2021$. 
[43] Q. Q. Liu, H. Liu, Z. G. He et al., "Differential gene and lncRNA expression in the lower thoracic spinal cord following ischemia/reperfusion-induced acute kidney injury in rats," Oncotarget, vol. 8, no. 32, pp. 53465-53481, 2017.

[44] B. W. Liu, Z. X. Li, Z. G. He, C. Liu, J. Xiong, and H. B. Xiang, "Altered expression of target genes of spinal cord in different itch models compared with capsaicin assessed by RT-qPCR validation," Oncotarget, vol. 8, no. 43, pp. 74423-74433, 2017.

[45] M. I. O. Milanez, E. E. Nishi, A. A. Rocha, C. T. Bergamaschi, and R. R. Campos, "Interaction between angiotensin II and GABA in the spinal cord regulates sympathetic vasomotor activity in Goldblatt hypertension," Neuroscience Letters, vol. 728, p. 134976, 2020.

[46] B. R. Bowman, P. Bokiniec, S. McMullan, A. K. Goodchild, and P. G. R. Burke, "Somatostatin 2 receptors in the spinal cord tonically restrain thermogenic, cardiac and other sympathetic outflows," Frontiers in Neuroscience, vol. 13, 2019.

[47] S. A. Deuchars, "How sympathetic are your spinal cord circuits?," Experimental Physiology., vol. 100, no. 4, pp. 365371, 2015.

[48] P. Pilowsky, I. J. Llewellyn-Smith, L. Arnolda, J. Minson, and J. Chalmers, "Intracellular recording from sympathetic preganglionic neurons in cat lumbar spinal cord," Brain Research., vol. 656, no. 2, pp. 319-328, 1994.

[49] Y.-Y. Poon, C.-Y. Tsai, C.-D. Cheng, A. Y. W. Chang, and S. H. H. Chan, "Endogenous nitric oxide derived from NOS I or II in thoracic spinal cord exerts opposing tonic modulation on sympathetic vasomotor tone via disparate mechanisms in anesthetized rats," American Journal of Physiology-Heart and Circulatory Physiology., vol. 311, no. 3, pp. H555-H562, 2016.

[50] K. C. Cowley, "A new conceptual framework for the integrated neural control of locomotor and sympathetic function: implications for exercise after spinal cord injury," Applied Physiology Nutrition and Metabolism., vol. 43, no. 11, pp. 11401150, 2018.

[51] S. Wang, X. Zhou, B. Huang et al., "Spinal cord stimulation suppresses atrial fibrillation by inhibiting autonomic remodeling," Heart Rhythm, vol. 13, no. 1, pp. 274-281, 2016.

[52] X. Ding, J. L. Ardell, F. Hua et al., "Modulation of cardiac ischemia-sensitive afferent neuron signaling by preemptive C2 spinal cord stimulation: effect on substance P release from rat spinal cord," American Journal of Physiology-Regulatory, Integrative and Comparative Physiology, vol. I, no. 294, pp. R93-R101, 2008.

[53] S. Wang, X. Zhou, B. Huang et al., "Spinal cord stimulation protects against ventricular arrhythmias by suppressing left stellate ganglion neural activity in an acute myocardial infarction canine model," Heart Rhythm, vol. 12, no. 7, pp. 16281635, 2015.

[54] C. Zhu, P. Hanna, P. S. Rajendran, and K. Shivkumar, "Neuromodulation for ventricular tachycardia and atrial fibrillation: a clinical scenario-based review," JACC: Clinical Electrophysiology, vol. 5, no. 8, pp. 881-896, 2019.

[55] N. H. Waldron, M. Fudim, J. P. Mathew, and J. P. Piccini, "Neuromodulation for the treatment of heart rhythm disorders," JACC: Basic to Translational Science, vol. 4, no. 4, pp. 546-562, 2019.

[56] B. M. Xu, L. Xiao, C. M. Kang et al., "LncRNA AC096664.3/PPAR- $\gamma / A B C G 1-d e p e n d e n t$ signal transduction pathway contributes to the regulation of cholesterol homeostasis," Journal of Cellular Biochemistry, vol. 120, no. 8, pp. 13775-13782, 2019.
[57] C. Cai, H. J. Zhu, X. T. Ning et al., "LncRNA ENST0000 0602558.1 regulates ABCG1 expression and cholesterol efflux from vascular smooth muscle cells through a p65-dependent pathway," Atherosclerosis, vol. 285, pp. 31-39, 2019.

[58] L. Li, L. Y. Wang, H. F. Li et al., "Characterization of LncRNA expression profile and identification of novel LncRNA biomarkers to diagnose coronary artery disease," Atherosclerosis, vol. 275, pp. 359-367, 2018.

[59] A. F. Gabriel and M. C. Costa, Eds.F. J. Enguita, "Interactions among regulatory non-coding RNAs involved in cardiovascular diseases," Advances in Experimental Medicine and Biology, A. F. Gabriel and M. C. Costa, Eds., vol. 1229, pp. 79-104, 2020.

[60] B. W. Li, Y. Z. Li, L. G. Hu et al., "Role of circular RNAs in the pathogenesis of cardiovascular disease," Journal of Cardiovascular Translational Research, vol. 13, no. 4, pp. 572-583, 2020.

[61] M. Y. Li, W. Ding, T. Sun et al., "Biogenesis of circular RNAs and their roles in cardiovascular development and pathology," The FEBS Journal, vol. 285, no. 2, pp. 220-232, 2018.

[62] Q. L. Zhou, Z. Zhang, Y. Bei, G. Li, and T. Wang, "Circular RNAs as novel biomarkers for cardiovascular diseases," in Circular RNAs. Advances in Experimental Medicine and Biology, Vol 1087, J. Xiao, Ed., pp. 159-170, Springer, Singapore, 2018.

[63] F. F. Li, X. Zhou, S. F. Chu, and N. H. Chen, "Inhibition of CKLF1 ameliorates hepatic ischemia-reperfusion injury via MAPK pathway," Cytokine, vol. 141, p. 7, 2021.

[64] X. X. Wan, K. Belanger, S. G. Widen, M. N. Kuyurncu-Martinez, and N. J. Garg, "Genes of the cGMP-PKG-Ca2+ signaling pathway are alternatively spliced in cardiomyopathy: role of RBFOX2," Biochimica et Biophysica Acta-Molecular Basis of Disease, vol. 1866, no. 3, p. 165620, 2020.

[65] Y. Q. Liu, S. J. Oh, K. H. Chang, Y. G. Kim, and M. Y. Lee, "Antiplatelet effect of AMP-activated protein kinase activator and its potentiation by the phosphodiesterase inhibitor dipyridamole," Biochemical Pharmacology, vol. 86, no. 7, pp. 914925, 2013.

[66] S. Charles and J. Natarajan, "Two way network construction and analysis of mRNA, miRNA and lncRNA reveals critical regulators and regulatory modules in cardiovascular diseases," Genes \& Genomics, vol. 42, no. 8, pp. 855-867, 2020.

[67] R. Kreutz, J. Ipe, K. Kassab et al., "P741 next generation miRNA sequencing and changes in coagulation measured by thrombelastography (TEG) in patients with cardiovascular disease," European Heart Journal, vol. 40, no. 1, pp. 346-346, 2019.

[68] W. Zhu, H. L. Wang, G. C. Sartor et al., "RNA sequencing analyses reveal eight miRNA/mRNA pairs that may mediate the effects of cocaine in cardiovascular diseases," Circulation, vol. 134, 2016.

[69] G. Wang, X. Fang, M. Han, X. Wang, and Q. Huang, "MicroRNA-493-5p promotes apoptosis and suppresses proliferation and invasion in liver cancer cells by targeting VAMP2," International Journal of Molecular Medicine, vol. 41, no. 3, pp. 1740-1748, 2018.

[70] T. Harel-Adar, T. Ben Mordechai, Y. Amsalem, M. S. Feinberg, J. Leor, and S. Cohen, "Modulation of cardiac macrophages by phosphatidylserine-presenting liposomes improves infarct repair," Proceedings of the National Academy of Sciences of the United States of America, vol. 108, no. 5, pp. 1827-1832, 2011.

[71] J. H. Rosenberg, J. H. Werner, M. J. Moulton, and D. K. Agrawal, "Current modalities and mechanisms underlying 
cardioprotection by ischemic conditioning," Journal of Cardiovascular Translational Research, vol. 11, no. 4, pp. 292-307, 2018.

[72] B. Z. Simkhovich, K. Przyklenk, and R. A. Kloner, "Role of protein kinase $\mathrm{C}$ in ischemic "conditioning": from first evidence to current perspectives," Journal of Cardiovascular Pharmacology and Therapeutics, vol. 18, no. 6, pp. 525-532, 2013.

[73] R. Gupta, L. Liu, X. Zhang et al., "IL-10 provides cardioprotection in diabetic myocardial infarction via upregulation of Heme clearance pathways," Insight, vol. 5, no. 17, 2020.

[74] D. Schumacher, A. Curaj, M. Staudt et al., "Phosphatidylserine supplementation as a novel strategy for reducing myocardial infarct size and preventing adverse left ventricular remodeling," International Journal of Molecular Sciences, vol. 22, no. 9, p. 4401, 2021.

[75] M. Bujak, M. Dobaczewski, C. Gonzalez-Quesada et al., "Induction of the CXC chemokine interferon-gammainducible protein 10 regulates the reparative response following myocardial infarction," Circulation Research, vol. 105, no. 10, pp. 973-983, 2009.

[76] M. Cavalera and N. G. Frangogiannis, "Targeting the chemokines in cardiac repair," Current Pharmaceutical Design, vol. 20, no. 12, pp. 1971-1979, 2014.

[77] B. Chen and N. G. Frangogiannis, "Chemokines in myocardial infarction," Journal of Cardiovascular Translational Research, vol. 14, no. 1, pp. 35-52, 2021.

[78] W. Xian, J. Wu, Q. Li et al., "CXCR3 alleviates renal ischemiareperfusion injury via increase of Tregs," Molecular Medicine Reports, vol. 24, no. 1, 2021.

[79] X. Jiang, X. Wu, F. Chen et al., "The profiles and networks of miRNA, lncRNA, mRNA, and circRNA in benzo(a)pyrenetransformed bronchial epithelial cells," Journal of Toxicological Sciences, vol. 43, no. 4, pp. 281-289, 2018.

[80] Q. Xu, X. Jia, Q. Wu et al., "Esomeprazole affects the proliferation, metastasis, apoptosis and chemosensitivity of gastric cancer cells by regulating lncRNA/circRNA-miRNA-mRNA ceRNA networks," Oncology Letters, vol. 20, 2020.

[81] K. Hu, C. He, X. Sun et al., "Integrated study of circRNA, lncRNA, miRNA, and mRNA networks in mediating the effects of testicular heat exposure," Cell and Tissue Research, p. 2021, 2021.

[82] Y. Cheng, Y. Su, S. Wang et al., "Identification of circRNAlncRNA-miRNA-mRNA competitive endogenous RNA network as novel prognostic markers for acute myeloid leukemia," Genes, vol. 11, no. 8, p. 868, 2020.

[83] C. Li, Z. Wang, J. Zhang et al., "Crosstalk of mRNA, miRNA, lncRNA, and circRNA and their regulatory pattern in pulmonary fibrosis," Molecular Therapy-Nucleic Acids, vol. 18, pp. 204-218, 2019.

[84] Y. Huang, "The novel regulatory role of lncRNA-miRNAmRNA axis in cardiovascular diseases," Journal of Cellular and Molecular Medicine, vol. 22, no. 12, pp. 5768-5775, 2018.

[85] M. Li, L. W. Duan, Y. X. Li, and B. Liu, "Long noncoding RNA/circular noncoding RNA-miRNA-mRNA axes in cardiovascular diseases," Life Sciences, vol. 233, p. 116440, 2019.
[86] W. Xiong, M. R. Yao, Y. Q. Yang, Y. Qu, and J. Q. Qian, "Implication of regulatory networks of long noncoding RNA/circular RNA-miRNA-mRNA in diabetic cardiovascular diseases," Epigenomics, vol. 12, no. 21, pp. 1929-1947, 2020.

[87] J. Wang, J. Yin, X. Wang et al., "Changing expression profiles of mRNA, lncRNA, circRNA, and miRNA in lung tissue reveal the pathophysiological of bronchopulmonary dysplasia (BPD) in mouse model," Journal of Cellular Biochemistry, vol. 120, no. 6, pp. 9369-9380, 2019. 